\title{
Spacetime diffeomorphisms as matter fields
}

Cite as: J. Math. Phys. 61, 111508 (2020); https://doi.org/10.1063/1.5140425

Submitted: 27 November 2019 . Accepted: 31 October 2020 . Published Online: 17 November 2020

Matteo Capoferri, and Dmitri Vassiliev

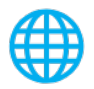

\section{ARTICLES YOU MAY BE INTERESTED IN}

Structures of (supersymmetric) classical W-algebras

Journal of Mathematical Physics 61, 111701 (2020); https://doi.org/10.1063/5.0010006

Quantum magic squares: Dilations and their limitations

Journal of Mathematical Physics 61, 111704 (2020); https://doi.org/10.1063/5.0022344

Riemann problem for the 2D scalar conservation law involving linear fluxes with

discontinuous coefficients

Journal of Mathematical Physics 61, 111504 (2020); https://doi.org/10.1063/5.0022048

\section{Journal of}

Mathematical Physics

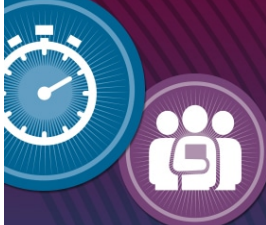

READY TO SUBMIT YOUR RESEARCH?

There are many reasons to publish with us. 


\title{
Spacetime diffeomorphisms as matter fields
}

\author{
Cite as: J. Math. Phys. 61, 111508 (2020); doi: 10.1063/1.5140425 \\ Submitted: 27 November 2019 • Accepted: 31 October 2020 • \\ Published Online: 17 November 2020
}

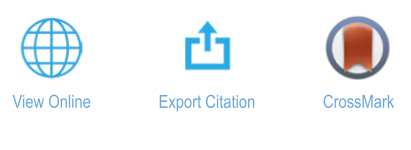

Matteo Capoferri ${ }^{a)}$ (D) and Dmitri Vassiliev ${ }^{\text {b) }}$ (D)

\begin{abstract}
AFFILIATIONS
Department of Mathematics, University College London, Gower Street, London WC1E 6BT, United Kingdom

a) Current address: School of Mathematics, Cardiff University, Senghennydd Road, Cardiff CF24 4AG, United Kingdom.

b) Author to whom correspondence should be addressed: d.vassiliev@ucl.ac.uk
\end{abstract}

\begin{abstract}
We work on a 4-manifold equipped with Lorentzian metric $g$ and consider a volume-preserving diffeomorphism that is the unknown quantity of our mathematical model. The diffeomorphism defines a second Lorentzian metric $h$, the pullback of $g$. Motivated by elasticity theory, we introduce a Lagrangian expressed algebraically (without differentiations) via our pair of metrics. Analysis of the resulting nonlinear field equations produces three main results. First, we show that for Ricci-flat manifolds, our linearized field equations are Maxwell's equations in the Lorenz gauge with exact current. Second, for Minkowski space, we construct explicit massless solutions of our nonlinear field equations; these come in two distinct types, right-handed and left-handed. Third, for Minkowski space, we construct explicit massive solutions of our nonlinear field equations; these contain a positive parameter that has the geometric meaning of quantum mechanical mass and a real parameter that may be interpreted as electric charge. In constructing explicit solutions of nonlinear field equations, we resort to group-theoretic ideas: we identify special four-dimensional subgroups of the Poincaré group and seek diffeomorphisms compatible with their action in a suitable sense.
\end{abstract}

Published under license by AIP Publishing. https://doi.org/10.1063/1.5140425

\section{INTRODUCTION}

In this paper, we propose a new mathematical model for a class of field theories in the Lorentzian setting. Inspired by the classical theory of elasticity (see, e.g., Refs. 1 and 2), we construct a Lagrangian out of a pair of metrics related by a spacetime diffeomorphism, which, in turn, represents the unknown of our model. The variation of our Lagrangian under the volume preservation condition produces a system of nonlinear partial differential equations, the field equations, whose analysis constitutes the main goal of this paper.

Our work possesses several elements of novelty. First, despite relying on ideas from Riemannian elasticity, our theory is fully Lorentzian in that it deals with diffeomorphisms of the whole spacetime into itself, giving a detailed account of the issues arising due to the indefinite signature. Second, our model incorporates a volume preservation condition into a theory of elasticity, leading to interesting mathematical consequences [see, e.g., (2.14) and (5.1)]. Third, we suggest new techniques for solving nonlinear partial differential equations (PDEs), ones of possibly broader relevance. Finally, our construction gives rise to solutions that appear to be physically meaningful, with potential applications in the realm of theoretical and particle physics.

For the case of Minkowski spacetime, we provide two classes of explicit solutions, massless and massive, which, at least at a formal level, offer a natural physical interpretation in terms of elementary particles, namely, neutrino/antineutrino and electron/positron. Our massive solution contains two free parameters. Even though these parameters can be interpreted as quantum mechanical mass and electric charge, our model does not allow for their values to be determined. We attribute this to a large number of symmetries implicitly present in our theory. One would hope that appropriate symmetry breaking could overcome this shortcoming of our mathematical model.

Our model is, effectively, a nonlinear version of Maxwell's theory. The only dimensional parameter is the speed of light: it is encoded in the Minkowski metric when we consider the case of flat spacetime. All other parameters are dimensionless and are contained in our Lagrangian. 
We develop our theory in dimension 4 and for pseudo-Riemannian manifolds of Lorentzian signature. In principle, neither assumption is necessary for its formulation. However, the physical conclusions we derive are specific to dimension $3+1$. In particular, dimension $3+1$ appears to be the lowest in which one observes propagating massless solutions.

This paper is structured as follows: In Sec. II, we present the mathematical formulation of our model. In Sec. III, we derive the corresponding nonlinear field equations, accounting for the volume preservation condition. Section IV is devoted to discussing the role of displacements and rotations; in particular, we perform a detailed analysis of the deformation gradient in terms of its Lorentzian polar decomposition. Section V contains our first main result: the linearized field equations and their connection with Maxwell's equations. For Ricci-flat Lorentzian manifolds, our model gives, in the linear approximation, Maxwell's equations in the Lorenz gauge with exact current. In Secs. VI and VII, we introduce the concept of homogeneous diffeomorphism and special subgroups of the Poincaré group, respectively. These represent the group-theoretic tools that lie at the foundation of our construction of solutions to nonlinear PDEs. Explicit solutions for Minkowski spacetime are presented in Secs. VIII and IX. Massless solutions described in Sec. VIII come in two types: right-handed and left-handed. Massive solutions described in Sec. IX contain two free parameters: a positive parameter that has the geometric meaning of quantum mechanical mass and a real parameter that may be interpreted as electric charge. Finally, in Secs. X and XI, we present a formal argument, showing that our massless and massive solutions can be associated with spinors satisfying the massless and massive Dirac equations, respectively. This constitutes the first step toward possible future applications of our model in theoretical and particle physics, e.g., in quantum electrodynamics (see also Ref. 3, where a volume preservation condition appears in a somewhat different setting). This paper is complemented by Appendixes A-D dealing with notation and auxiliary technical results.

\section{MATHEMATICAL MODEL}

Let $M$ be a connected 4-manifold. Local coordinates on $M$ will be denoted by $x=\left(x^{1}, x^{2}, x^{3}, x^{4}\right)$ or $y=\left(y^{1}, y^{2}, y^{3}, y^{4}\right)$.

We assume that our manifold $M$ is equipped with Lorentzian metric $g$ with signature +++- . Throughout this paper, the metric $g$ is assumed to be prescribed. $\operatorname{Diff}(M)$.

The unknown quantity in our mathematical model is a diffeomorphism $\varphi: M \rightarrow M$. We will denote the group of diffeomorphisms by

Let us introduce a new (perturbed) Lorentzian metric $h$ defined as the pullback of $g$ via $\varphi, h:=\varphi^{*} g$. In local coordinates, this new metric is written as follows: Take an arbitrary point $P \in M$ and choose local coordinates $x$ and $y$ in the neighborhoods of $P$ and $\varphi(P)$, respectively. Our diffeomorphism $\varphi$ can then be written locally as

$$
y=\varphi(x)
$$

The new metric tensor reads

$$
h_{\alpha \beta}(x):=g_{\mu v}(\varphi(x)) \frac{\partial \varphi^{\mu}}{\partial x^{\alpha}} \frac{\partial \varphi^{v}}{\partial x^{\beta}}
$$

The $g_{\mu \nu}$ in the RHS of (2.2) is the representation of the metric tensor $g$ in local coordinates $y$.

The following non-rigorous physical argument along the lines of Ref. 4 explains the geometric meaning of the tensor (2.2). Consider two points, $x$ and $x+\Delta x$. The interval (Lorentzian analog of "distance squared") between these two points is $g_{\alpha \beta}(x) \Delta x^{\alpha} \Delta x^{\beta}$. Our diffeomorphism maps $x$ and $x+\Delta x$ to $\varphi(x)$ and $\varphi(x+\Delta x) \approx \varphi(x)+\frac{\partial \varphi}{\partial x^{\alpha}} \Delta x^{\alpha}$, respectively. The interval between $\varphi(x)$ and $\varphi(x)+\frac{\partial \varphi}{\partial x^{\alpha}} \Delta x^{\alpha}$ is $g_{\mu v}(\varphi(x))\left(\frac{\partial \varphi^{\mu}}{\partial x^{\alpha}} \Delta x^{\alpha}\right)\left(\frac{\partial \varphi^{v}}{\partial x^{\beta}} \Delta x^{\beta}\right)$, which, in view of (2.2), can be rewritten concisely as $h_{\alpha \beta}(x) \Delta x^{\alpha} \Delta x^{\beta}$. Therefore, the metric $h$ describes the interval between points of the deformed continuum.

Having at our disposal two Lorentzian metrics, $g$ and $h$, we can now write down an action. To this end, let us first introduce some definitions.

Definition 2.1. The tensor

$$
S_{\beta}^{\alpha}(x):=\left[g^{\alpha \gamma}(x)\right]\left[h_{\gamma \beta}(x)\right]-\delta_{\beta}^{\alpha}
$$

is called strain.

The concept of strain tensor originates from the papers of Cauchy. 5,6

The strain tensor describes, pointwise, a linear map in the fibers of the tangent bundle,

$$
v^{\alpha} \mapsto S_{\beta}^{\alpha} v^{\beta}
$$

The algebraic motivation for the introduction of the map (2.4) is explained in Appendix B 1.

Let us now construct scalars out of a strain tensor. This can be done in many different ways but only four, at most, will be independent. An arbitrary scalar can be expressed, possibly in a nonlinear fashion, via the four chosen independent scalars. The obvious way of choosing four independent scalars is $\operatorname{tr}\left(S^{k}\right), k=1,2,3,4$, but such a choice is inconvenient as it would make subsequent calculations cumbersome. The most convenient choice of four scalar invariants is 


$$
\begin{aligned}
& e_{1}(\varphi):=\operatorname{tr} S, \\
& e_{2}(\varphi):=\frac{1}{2}\left[(\operatorname{tr} S)^{2}-\operatorname{tr}\left(S^{2}\right)\right], \\
& e_{3}(\varphi):=\operatorname{tr} \operatorname{adj} S, \\
& e_{4}(\varphi):=\operatorname{det} S
\end{aligned}
$$

Here, $t r$ is the matrix trace and adj is the operator of matrix adjugation from linear algebra.

The reasoning behind the particular choice $(2.5 \mathrm{a})-(2.5 \mathrm{~d})$ becomes clear if we rewrite these invariants in terms of the eigenvalues of strain. The strain tensor (2.3), viewed as a linear operator (2.4) acting in $\mathbb{C}^{4}$, has eigenvalues $\lambda_{k}, k=1,2,3,4$, enumerated with account of their algebraic multiplicity. Note that some eigenvalues may be complex in which case they come in complex conjugate pairs. It is easy to see that formulas $(2.5 \mathrm{a})-(2.5 \mathrm{~d})$ can be rewritten as

$$
\begin{aligned}
& e_{1}(\varphi)=\lambda_{1}+\lambda_{2}+\lambda_{3}+\lambda_{4}, \\
& e_{2}(\varphi)=\lambda_{1} \lambda_{2}+\lambda_{1} \lambda_{3}+\lambda_{1} \lambda_{4}+\lambda_{2} \lambda_{3}+\lambda_{2} \lambda_{4}+\lambda_{3} \lambda_{4}, \\
& e_{3}(\varphi)=\lambda_{1} \lambda_{2} \lambda_{3}+\lambda_{1} \lambda_{2} \lambda_{4}+\lambda_{1} \lambda_{3} \lambda_{4}+\lambda_{2} \lambda_{3} \lambda_{4}, \\
& e_{4}(\varphi)=\lambda_{1} \lambda_{2} \lambda_{3} \lambda_{4} .
\end{aligned}
$$

The advantage of choosing scalar invariants in this particular way is that the polynomials appearing in the right-hand sides of formulas (2.6a) $-(2.6 \mathrm{~d})$ are elementary symmetric polynomials.

Note that our scalars $e_{k}$ are spectral invariants: we are looking at quantities that are determined by the spectrum of the linear map (2.4). Our definition of scalar invariants is similar to that in Ref. 7 (3.56), the only difference being that we have four scalar invariants instead of three-a consequence of us adopting a four-dimensional relativistic approach.

Our action then is

$$
\mathcal{J}(\varphi):=\int_{M} \mathcal{L}\left(e_{1}(\varphi), e_{2}(\varphi), e_{3}(\varphi), e_{4}(\varphi)\right) \sqrt{-\operatorname{det} g_{\mu \nu}(x)} d x
$$

where $\mathcal{L}$ is some prescribed smooth real-valued function of four real variables such that $\mathcal{L}(0,0,0,0)=0$ and $d x:=d x^{1} d x^{2} d x^{3} d x^{4}$. Variation of (2.7) with respect to the unknown diffeomorphism $\varphi \in \operatorname{Diff}(M)$ generates field equations, which can be thought of as a Lorentzian version of nonlinear elasticity.

The physical assumptions underlying our choice of action (2.7) are isotropy and homogeneity of our four-dimensional continuum. Isotropy is expressed mathematically in that the integrand $\mathcal{L}$ in (2.7) is a symmetric function of the eigenvalues of the map (2.4). Homogeneity is expressed mathematically in that the integrand $\mathcal{L}$ in (2.7) does not depend explicitly on $x$.

Two important examples of Lagrangians are given below.

Example 2.2 (Linear Lagrangian). The unique, up to rescaling, Lagrangian linear in strain is

$$
\mathcal{L}\left(e_{1}, e_{2}, e_{3}, e_{4}\right)=e_{1} .
$$

This is the action of a harmonic map (see Refs. 8 and 9), the only difference being that in our paper, the metric is assumed to have Lorentzian signature.

Example 2.3 (Quadratic Lagrangian). The general form of a Lagrangian quadratic (homogeneous of degree two) in strain is

$$
\mathcal{L}\left(e_{1}, e_{2}, e_{3}, e_{4}\right)=\alpha\left(e_{1}\right)^{2}+\beta e_{2}
$$

where $\alpha, \beta \in \mathbb{R}$ are parameters. In the three-dimensional Riemannian setting, the above Lagrangian is used in the theory of elasticity: it describes a static isotropic homogeneous elastic continuum that is physically linear but geometrically nonlinear. The standard assumption in elasticity theory is

$$
\beta \neq 0 .
$$

Under the assumption (2.10), the Lagrangian (2.9) contains, up to rescaling, only one dimensionless parameter, $\alpha / \beta$. In elasticity theory, the parameters $\lambda=2 \alpha+\beta$ and $\mu=-\beta / 2$ are called Lamé parameters and the parameter $v=\frac{2 \alpha+\beta}{4 \alpha+\beta}$ is called Poisson's ratio.

Remark 2.4. Our mathematical model does not involve the concepts of connection and curvature. Moreover, it is easy to see that if the unperturbed metric $g$ is flat, then the perturbed metric $h$ is flat as well. Our model is different from those commonly used in theories of bimetric gravity, ${ }^{10-13}$ even though the mathematical formalism is quite similar. 
Field equations for the action (2.7) are not the equations that we will be studying. We choose to impose, in addition, the volume preservation constraint,

$$
\operatorname{det} g_{\alpha \beta}(x)=\operatorname{det} h_{\mu v}(x)
$$

In other words, we choose to restrict our analysis to the subgroup of volume-preserving diffeomorphisms $\operatorname{Diff}(M) \subset \operatorname{Diff}(M)$. $\operatorname{Here}$,

$$
\rho(x):=\sqrt{-\operatorname{det} g_{\alpha \beta}(x)}
$$

is the Lorentzian density of the unperturbed metric.

The condition for a diffeomorphism to be volume preserving reads, locally,

$$
\rho(x)=\rho(\varphi(x))\left|\operatorname{det}\left(\frac{\partial \varphi^{\alpha}}{\partial x^{\beta}}\right)\right| .
$$

The $\rho$ in the LHS of (2.13) is the representation of the density $\rho$ in local coordinates $x$, whereas the $\rho$ in the RHS of (2.13) is the representation of the density $\rho$ in local coordinates $y$ [see (2.1)].

In a spectral-theoretic fashion, the volume preservation constraint (2.11) can be equivalently rewritten as

$$
e_{1}(\varphi)+e_{2}(\varphi)+e_{3}(\varphi)+e_{4}(\varphi)=0
$$

Indeed, formula (2.3) implies that condition (2.11) is equivalent to $\operatorname{det}(S+\mathrm{Id})=1$. The elementary identity from linear algebra

$$
\operatorname{det}(S+\mathrm{Id})=1+\operatorname{tr} S+\frac{1}{2}\left[(\operatorname{tr} S)^{2}-\operatorname{tr}\left(S^{2}\right)\right]+\operatorname{tr} \operatorname{adj} S+\operatorname{det} S
$$

and $(2.5 \mathrm{a})-(2.5 \mathrm{~d})$ then give us $(2.14)$.

Formula (2.14) allows us to express one of the four scalar invariants via the other three. It is convenient to express $e_{1}$ via $e_{2}, e_{3}$, and $e_{4}$. Then, our action (2.7) takes the form

$$
J(\varphi)=\int_{M} L\left(e_{2}(\varphi), e_{3}(\varphi), e_{4}(\varphi)\right) \rho(x) d x
$$

where

$$
L\left(e_{2}, e_{3}, e_{4}\right)=\mathcal{L}\left(-e_{2}-e_{3}-e_{4}, e_{2}, e_{3}, e_{4}\right)
$$

Our mathematical model is formulated as follows: vary the action (2.15) over volume preserving diffeomorphisms Diff $(M)$ and seek critical points. The $L$ appearing in formula (2.15) is some prescribed smooth real-valued function of three real variables, which characterizes the physical properties of our four-dimensional isotropic homogeneous continuum.

We shall now impose two conditions on the choice of the Lagrangian $L$.

Condition 1. We assume that

$$
\left.\frac{\partial L}{\partial e_{2}}\right|_{e_{2}=e_{3}=e_{4}=0} \neq 0,
$$

which is the minimal non-degeneracy condition. This will be required in Sec. V where we will show that in a Ricci-flat spacetime, our linearized field equations reduce to Maxwell's equations. Without loss of generality, we assume further on that

$$
\left.\frac{\partial L}{\partial e_{2}}\right|_{e_{2}=e_{3}=e_{4}=0}=-1,
$$

which can always be achieved by rescaling.

Condition 2. We assume that the function of one variable $L\left(e_{2}, 0,0\right)$ has a critical point on the positive real axis,

$$
\left.\frac{\partial L}{\partial e_{2}}\right|_{e_{2}=c, e_{3}=e_{4}=0}=0 \text { for some } c>0 .
$$

This will be required in Sec. IX where we will construct explicit massive solutions of our nonlinear field equations in Minkowski spacetime. 
Example 2.5 (Examples 2.2 and 2.3 continued). For the Lagrangian (2.16) and (2.8), we get precisely (2.17b), whereas for the Lagrangian (2.16) and (2.9), we get

$$
\left.\frac{\partial L}{\partial e_{2}}\right|_{e_{2}=e_{3}=e_{4}=0}=\beta
$$

so condition (2.17a) is satisfied when we have (2.10).

As to condition (2.18), it is not satisfied for the Lagrangian (2.16) and (2.8), whereas for the Lagrangian (2.16) and (2.9), it is satisfied if and only if $\alpha \beta<0$.

\section{NONLINEAR FIELD EQUATIONS}

Recall that the action in our mathematical model is defined by formula (2.15). Our field equations are obtained by varying this action with respect to the unknown diffeomorphism $\varphi$ subject to the volume preservation constraint (2.11).

In order to write down the field equations, let us initially disregard the constraint (2.11) and argue along the lines of Chapter 8 (Ref. 14). In local coordinates, our action (2.15) can be written as

$$
J(\varphi)=\int f\left(x^{\alpha}, \varphi^{\beta}, \frac{\partial \varphi^{\gamma}}{\partial x^{\kappa}}\right) \rho(x) d x,
$$

where $\varphi^{\beta}$ is the local representation (2.1) of our diffeomorphism and $f$ is some function of $x, \varphi(x)$ and the first partial derivatives of $\varphi(x)$. We vary $\varphi(x)$ as

$$
\varphi^{\beta}(x) \mapsto \varphi^{\beta}(x)+\Delta \varphi^{\beta}(x)
$$

where $\Delta \varphi^{\beta}(x)$ is a small smooth perturbation with small compact support. Standard variational arguments involving integration by parts give us the variation of (3.1) in the form

$$
\Delta J(\varphi)=\int E_{\lambda}\left(x^{\alpha}, \varphi^{\beta}, \frac{\partial \varphi^{\gamma}}{\partial x^{\kappa}}, \frac{\partial^{2} \varphi^{\sigma}}{\partial x^{\mu} \partial x^{\nu}}\right) \Delta \varphi^{\lambda} \rho(x) d x .
$$

The quantity $E_{\lambda}$ appearing in the RHS of (3.3) is a two-point tensor: it behaves as a scalar under changes of local coordinates $x$ and as a covector under changes of local coordinates $y$. Hence,

$$
\varphi \mapsto E_{\lambda}\left(x^{\alpha}, \varphi^{\beta}, \frac{\partial \varphi^{\gamma}}{\partial x^{\kappa}}, \frac{\partial^{2} \varphi^{\sigma}}{\partial x^{\mu} \partial x^{\nu}}\right)
$$

is an invariantly defined map from diffeomorphisms to covector fields.

We write the RHS of (3.4) in concise form as $E(\varphi)$. Thus, the field equations for the unconstrained action (2.15) read

$$
E(\varphi)=0 .
$$

This is a system of four nonlinear second order partial differential equations for four unknowns, the functions $\varphi^{\alpha}(x), \alpha=1,2,3,4$, appearing in the local representation (2.1) of our diffeomorphism $\varphi$.

A detailed algorithm for the construction of the nonlinear differential operator $E$ is provided in Appendix D. However, we do not need the explicit form of $E$ for our purposes. Even when we will be writing particular solutions of our nonlinear field equations (see Secs. VIII and IX), we will do this without using the explicit form of the operator $E$.

Remark 3.1. Straightforward analysis shows that the identity map is a solution of (3.5). Furthermore, any isometry from $(M, g)$ to itself is a solution.

Let us now incorporate the volume preservation constraint (2.11) by adding to our original action (2.15) the term

$$
K(\varphi, p):=\int[p(\varphi(x))]\left[\rho_{\varphi}(x)-\rho(x)\right] d x
$$

where $\rho_{\varphi}(x):=\sqrt{-\operatorname{det} h_{\alpha \beta}(x)}$ and $p: M \rightarrow \mathbb{R}$ is an additional unknown scalar function playing the role of a Lagrange multiplier. The function $p$ can be interpreted as pressure (cf. Ref. 15).

We will now vary our diffeomorphism as in (3.2).

Lemma 3.2. The formula for the variation of the functional (3.6) reads

$$
\Delta K(\varphi, p)=-\int\left[\frac{\partial p}{\partial y^{\alpha}}(\varphi(x))\right]\left[\Delta \varphi^{\alpha}(x)\right][\rho(x)] d x .
$$


Proof. Observe that the diffeomorphism $\varphi$ appears in formula (3.6) twice, so

$$
\Delta K(\varphi, p)=\Delta K_{1}(\varphi, p)+\Delta K_{2}(\varphi, p)
$$

where

$$
\begin{gathered}
K_{1}(\varphi, p):=\int[p(\varphi(x))]\left[\rho_{\varphi}(x)-\rho(x)\right] d x, \\
K_{2}(\varphi, p):=\int[p(\varphi(x))]\left[\rho_{\varphi}(x)\right] d x,
\end{gathered}
$$

the bold script indicating that this particular occurrence of $\varphi$ is not subject to variation (3.2).

Variation of (3.9) gives us

$$
\Delta K_{1}(\varphi, p)=\int\left[\frac{\partial p}{\partial y^{\alpha}}(\varphi(x))\right]\left[\Delta \varphi^{\alpha}(x)\right]\left[\rho_{\varphi}(x)-\rho(x)\right] d x .
$$

In order to calculate the variation of (3.10), we switch from local coordinates $x$ to local coordinates $y$ in accordance with $y=\varphi(x)$. Formula (3.10) now reads

$$
K_{2}(\varphi, p)=\int[p(y)]\left[\mu_{\varphi}(y)\right] d y^{1} d y^{2} d y^{3} d y^{4}
$$

where $\mu_{\varphi}$ is the representation of the density $\rho_{\varphi}$ in local coordinates $y$. A straightforward calculation [see also (4.10f) and (4.12)] shows that

$$
\Delta \mu_{\varphi}(y)=\frac{\partial\left(\left[\mu_{\varphi}(y)\right]\left[\Delta \varphi^{\alpha}\left(\varphi^{-1}(y)\right)\right]\right)}{\partial y^{\alpha}} .
$$

Indeed, Jacobi's formula tells us that for any invertible matrix $A$, we have

$$
\Delta(\operatorname{det} A)=(\operatorname{det} A) \operatorname{tr}\left(A^{-1} \Delta A\right) .
$$

Applying (3.14) to the density $\rho_{\varphi}(x)$, we obtain

$$
\Delta \rho_{\varphi}(x)=\frac{1}{2} \rho_{\varphi}(x) h^{\alpha \beta}(x) \Delta h_{\alpha \beta}(x)
$$

The variation of (2.2) reads

$$
\Delta h_{\alpha \beta}(x)=\frac{\partial g_{\mu v}}{\partial y^{\gamma}}(\varphi(x)) \Delta \varphi^{\gamma} \frac{\partial \varphi^{\mu}}{\partial x^{\alpha}} \frac{\partial \varphi^{v}}{\partial x^{\beta}}+g_{\mu v}(\varphi(x)) \frac{\partial \Delta \varphi^{\mu}}{\partial x^{\alpha}} \frac{\partial \varphi^{v}}{\partial x^{\beta}}+g_{\mu v}(\varphi(x)) \frac{\partial \varphi^{\mu}}{\partial x^{\alpha}} \frac{\partial \Delta \varphi^{v}}{\partial x^{\beta}}
$$

Formula (2.2) implies that the inverse metric tensor $h^{\alpha \beta}$ can be written as

$$
h^{\alpha \beta}(x)=g^{\mu \nu}(\varphi(x)) \psi_{\mu}{ }^{\alpha} \psi_{v}{ }^{\beta},
$$

where the two-point tensor $\psi_{\alpha}{ }^{\beta}$ is defined by the identity

$$
\psi_{\alpha}^{\beta}(x) \frac{\partial \varphi^{\gamma}}{\partial x^{\beta}}(x)=\delta_{\alpha}^{\gamma} .
$$

Substituting (3.16) and (3.17) into (3.15) and using (3.18), we get

$$
\Delta \rho_{\varphi}(x)=\rho_{\varphi}(x)\left[\frac{1}{2} g^{\mu \nu}(\varphi(x)) \frac{\partial g_{\mu v}}{\partial y^{\gamma}}(\varphi(x)) \Delta \varphi^{\gamma}+\frac{\partial \Delta \varphi^{\mu}}{\partial y^{\mu}}(x)\right] .
$$

The above formula and the fact that

$$
\frac{\Delta \mu_{\varphi}}{\mu_{\varphi}}(\varphi(x))=\frac{\Delta \rho_{\varphi}}{\rho_{\varphi}}(x)
$$

imply (3.13). 
Substituting (3.13) into (3.12) and integrating by parts, we get

$$
\Delta K_{2}(\varphi, p)=-\int\left[\frac{\partial p}{\partial y^{\alpha}}(y)\right]\left[\Delta \varphi^{\alpha}\left(\varphi^{-1}(y)\right)\right]\left[\mu_{\varphi}(y)\right] d y^{1} d y^{2} d y^{3} d y^{4}
$$

It remains only to switch back from local coordinates $y$ to local coordinates $x$. Formula (3.19) becomes

$$
\Delta K_{2}(\varphi, p)=-\int\left[\frac{\partial p}{\partial y^{\alpha}}(\varphi(x))\right]\left[\Delta \varphi^{\alpha}(x)\right]\left[\rho_{\varphi}(x)\right] d x .
$$

Substituting (3.11) and (3.20) into (3.8), we arrive at (3.7).

Lemma 3.2 tells us that the field equations for the constrained action (2.15) read

$$
E(\varphi)-\mathrm{d} p=0,
$$

where $\mathrm{d} p$ is the gradient of pressure $p$. Equation (3.21) has to be supplemented by the volume preservation condition (2.11).

The term $\mathrm{d} p$ appearing in formula (3.21) can be written in local coordinates as

$$
(\mathrm{d} p)_{\alpha}(x)=\psi_{\alpha}^{\beta}(x) \frac{\partial(p \circ \varphi)}{\partial x^{\beta}}(x)
$$

where the two-point tensor $\psi_{\alpha}{ }^{\beta}$ was defined in (3.18).

Formulas (3.21) and (2.11) give us a system of five partial differential equations for five unknowns, the functions $\varphi^{\alpha}(x), \alpha=1,2,3,4$, appearing in the local representation (2.1) of our diffeomorphism $\varphi$ and the scalar field $(p \circ \varphi)(x)$.

\section{DISPLACEMENTS AND ROTATIONS}

Suppose that our diffeomorphism $\varphi: M \rightarrow M$ is sufficiently close to the identity map. Then, it can be described by a vector field of displacements $A$. This vector field can be equivalently defined in two different ways.

Take an arbitrary point $P \in M$ and let $\Omega \subset M$ be a normal, with respect to $g$, neighborhood of $P$. As $\varphi$ is close to the identity map, we can assume, without loss of generality, that $\varphi(P) \in \Omega$. Let $\gamma:[0,1] \rightarrow \Omega$ be the unique shortest geodesic, with respect to $g$, connecting $P$ and $\varphi(P)$, so that $\gamma(0)=P$ and $\gamma(1)=\varphi(P)$. Furthermore, let us parameterize our geodesic in such a way that $\gamma(\tau)$ is a solution of the equation

$$
\ddot{\gamma}^{\lambda}+\Gamma_{\mu \nu}^{\lambda} \dot{\gamma}^{\mu} \dot{\gamma}^{\nu}=0
$$

where $\Gamma_{\mu \nu}^{\lambda}$ are Christoffel symbols and the dot stands for differentiation in $\tau$. Then,

$$
A(P):=\dot{\gamma}(0)
$$

Alternatively, let $W(P, Q)$ be the Ruse-Synge world function (Ref. 16, Chap. II, Sec. 1) with respect to $g$. Here, $P, Q \in M$ are assumed to be sufficiently close. Let $W^{\prime}(P, Q):=\left.\operatorname{grad}_{x} W(x, Q)\right|_{x=P}$ be the gradient of the world function with respect to the first variable. Then,

$$
A^{b}(P):=-W^{\prime}(P, \varphi(P))
$$

In formula (4.1), $A$ is a vector, whereas in formula (4.2), $A^{b}$ is a covector. Raising and lowering tensor indices via the metric $g$ turns one into the other (see Appendix A 1 for notation).

Working with a vector field of displacements $A$ rather than an abstract diffeomorphism $\varphi$ makes the physical interpretation clearer.

The field of displacements generates rotations. Describing these rotations mathematically is the subject of finite strain theory in continuum mechanics (Ref. 17, Sec. 2). In what follows, we present this construction in a version adapted to Lorentzian signature and curved spacetime.

Consider the quantity

$$
\frac{\partial \varphi^{v}}{\partial x^{\beta}}(x)
$$

The quantity (4.3) is a two-point tensor: it transforms as a covector under changes of local coordinates $x$ and as a vector under changes of local coordinates $y$. The two-point tensor (4.3) describes a linear map from $T_{P} M$ to $T_{\varphi(P)} M$,

$$
v^{\alpha} \mapsto \frac{\partial \varphi^{v}}{\partial x^{\beta}} v^{\beta}
$$


Let us now parallel transport (4.3), in the upper tensor index and with respect to the Levi-Civita connection associated with $g$, along the unique shortest geodesic from $\varphi(P)$ to $P$. This gives us a (one-point) $(1,1)$-tensor $D_{\beta}^{v}(x)$ known in continuum mechanics as the deformation gradient. In local coordinates, the deformation gradient is explicitly obtained as follows: Let us choose the same coordinates $x$ and $y$, and let

$$
\gamma:[0,1] \rightarrow M, \quad \gamma(0)=\varphi(P), \quad \gamma(1)=P
$$

be the unique geodesic connecting $\varphi(P)$ and $P$. This is, of course, the same geodesic $\gamma$ as in the beginning of this section, and only now we use a different parameterization, $\tau \mapsto s:=1-\tau$. Then, $D_{\beta}^{v}:=X_{\beta}^{v}(1)$, where $X_{\beta}^{v}(s)$ is the unique solution to the ordinary differential equation

$$
\frac{d X_{\beta}^{v}(s)}{d s}+X_{\beta}^{\alpha}(s) \Gamma_{\alpha \mu}^{v}(\gamma(s)) \frac{d \gamma^{\mu}(s)}{d s}=0
$$

subject to the initial condition $X_{\beta}^{v}(0)=\frac{\partial \varphi^{v}}{\partial x^{\beta}}$. The deformation gradient describes, pointwise, a non-degenerate linear map in the fibers of the tangent bundle,

$$
v^{\alpha} \mapsto D_{\beta}^{v} v^{\beta}
$$

Moreover, formula (2.2) can now be rewritten as

$$
h_{\alpha \beta}(x)=\left[D_{\alpha}^{\mu}(x)\right]\left[g_{\mu v}(x)\right]\left[D_{\beta}^{v}(x)\right]
$$

Remark 4.1. Even though, in general, there is no explicit closed formula relating the vector field of displacements $A$ and the deformation gradient $D$, there is a connection between the two expressed by formula (4.10a). In special circumstances, such a connection can be made sharper, for instance, when working in Minkowski space [see formula (8.3)].

Furthermore, we assume that the linear map (4.5) is sufficiently close to the identity. The issue at hand is to decompose (4.5) into a composition of a stretching map and a rotation map. This is achieved by means of the polar decomposition. The concept of polar decomposition is standard in linear algebra, only now it has to be adapted to Lorentzian signature. Some work in this direction was done in Refs. 18 and 19.

Definition 4.2. We call a linear map $v^{\alpha} \mapsto B^{\alpha}{ }_{\beta} v^{\beta}$ Lorentz-symmetric if $g_{\alpha \gamma} B_{\beta}^{\gamma}=g_{\beta \gamma} B_{\alpha}^{\gamma}$, Lorentz-antisymmetric if $g_{\alpha \gamma} B_{\beta}^{\gamma}=-g_{\beta \gamma} B^{\gamma}$, and Lorentz-orthogonal if $B^{\mu}{ }_{\alpha \mu \nu} B_{\beta}^{v}=g_{\alpha \beta}$.

Any linear map (4.5) sufficiently close to the identity can be uniquely decomposed as

$$
D_{\beta}^{\alpha}=U_{\gamma}^{\alpha} V_{\beta}^{\gamma} \text {, }
$$

where $U$ is Lorentz-orthogonal and $V$ is Lorentz-symmetric and close to the identity.

Recall that the standard polar decomposition theorem tells us that an invertible real matrix $K$ can be written as the product of an orthogonal matrix $U_{K}$ and a symmetric matrix $V_{K}, K=U_{K} V_{K}$, where these matrices are given by the explicit formulas,

$$
V_{K}=\left(K^{T} K\right)^{1 / 2} \text { and } \quad U_{K}=K\left(K^{T} K\right)^{-1 / 2} \text {. }
$$

The existence of polar decomposition (4.7) can be established, for example, by using the power series expansion for the function $\sqrt{1+z}$ with $z=g^{\alpha \gamma} D^{\mu}{ }_{\gamma} g_{\mu \nu} D^{v}{ }_{\beta}-\delta^{\alpha}{ }_{\beta}$ and arguing along the lines of the classical proof. Indeed, the quantity $D^{\mu}{ }_{\gamma} g_{\mu \nu} D^{v}{ }_{\beta}$ plays now the role of $K^{T} K$ [see also (4.6)], and the fact that (4.5) is sufficiently close to the identity ensures that the series converges and that the outcome is invertible.

In the setting of classical elasticity theory (Riemannian signature), the tensor $V$ appearing in formula (4.7) is called the right stretch tensor (see Ref. 17, p. 53).

Formula (4.7) and the fact that $D$ and $V$ are close to the identity imply that $U$ is close to the identity as well. Therefore, $U$ can be uniquely represented as

$$
U=e^{F} \text {, }
$$

where $F$ is Lorentz-antisymmetric and small. The tensor $F$ can be recovered from the tensor $U$ by using the power series expansion for the function $\ln (1+z)$ with $z=U^{\alpha}{ }_{\beta}-\delta^{\alpha}{ }_{\beta}$.

Applying the above procedure to the deformation gradient, we arrive at a Lorentz-antisymmetric $(1,1)$-tensor $F_{\beta}^{\alpha}(x)$. Lowering the first tensor index via $g$, we get a covariant antisymmetric tensor $F_{\alpha \beta}(x)$, which can be viewed as a 2 -form. We call it the rotation 2 -form.

Substituting (4.7) into (4.6), we get

$$
h_{\alpha \beta}(x)=\left[V_{\alpha}^{\mu}(x)\right]\left[g_{\mu v}(x)\right]\left[V_{\beta}^{v}(x)\right]
$$

Remark 4.3. The order of indices in our polar decomposition (4.7) is important. Had we done the polar decomposition the other way round, i.e., as $D_{\beta}^{\alpha}=V_{\gamma}^{\alpha} U_{\beta}^{\gamma}$, we would not have gotten (4.9). 
Remark 4.4. The assumption that our map (4.5) is sufficiently close to the identity warrants further clarification. As explained earlier in this section, this assumption is sufficient to ensure the existence of a Lorentzian polar decomposition. However, it is, in general, not necessary, as one can see by considering the linear map (4.5) with

$$
D_{\beta}^{v}=\left(\begin{array}{cc}
9 & 0 \\
0 & -99
\end{array}\right)
$$

in two-dimensional Krein space $\left[g_{\kappa \lambda}=\operatorname{diag}(1,-1)\right]$, which clearly admits a Lorentzian polar decomposition. The issue at hand is that necessary and sufficient conditions for the existence of polar decomposition of matrices in indefinite inner product spaces are not available in the literature. While this is a very interesting problem in linear algebra, addressing it in the current paper would lead us astray. Therefore, we decided to impose what we view as a reasonable sufficient condition, with the plan to deal with the general theory of Lorentzian polar decomposition elsewhere.

Formula (4.9) tells us that rotations do not appear explicitly in our mathematical model. In other words, the physics described by our action (2.15) does not feel rotations. However, we will still have to consider rotations later on in this paper because they do not have a life of their own: rotations are generated by displacements (cf. Secs. X and XI).

The following lemma provides a list of formulas obtained by linearizing in A. Some of them will be used in Sec. V.

Lemma 4.5. We have

$$
\begin{aligned}
& D_{\alpha \beta}=g_{\alpha \beta}+\nabla_{\beta} A_{\alpha}+O\left(|A|^{2}\right), \\
& V_{\alpha \beta}=g_{\alpha \beta}+\frac{1}{2}\left(\nabla_{\alpha} A_{\beta}+\nabla_{\beta} A_{\alpha}\right)+O\left(|A|^{2}\right), \\
& U_{\alpha \beta}=g_{\alpha \beta}-\frac{1}{2}\left(\nabla_{\alpha} A_{\beta}-\nabla_{\beta} A_{\alpha}\right)+O\left(|A|^{2}\right), \\
& F_{\alpha \beta}=-\frac{1}{2}\left(\nabla_{\alpha} A_{\beta}-\nabla_{\beta} A_{\alpha}\right)+O\left(|A|^{2}\right), \\
& S_{\alpha \beta}=\nabla_{\alpha} A_{\beta}+\nabla_{\beta} A_{\alpha}+O\left(|A|^{2}\right), \\
& \frac{\operatorname{det} h_{\kappa \lambda}}{\operatorname{det} g_{\mu \nu}}=1+2 \nabla_{\alpha} A^{\alpha}+O\left(|A|^{2}\right) .
\end{aligned}
$$

In the above lemma and further on, $\nabla$ is the Levi-Civita connection associated with $g$ and tensor indices are raised and lowered using the metric $g$. In particular, the tensor in the LHS of formula (4.10e) is our original strain tensor (2.3) but with the first tensor index lowered. Of course, we have $S_{\alpha \beta}=h_{\alpha \beta}-g_{\alpha \beta}$.

Note that formulas (4.10d) and (4.10f) can be equivalently rewritten without covariant derivatives using the identities

$$
\begin{gathered}
\nabla_{\alpha} A_{\beta}-\nabla_{\beta} A_{\alpha}=\partial_{\alpha} A_{\beta}-\partial_{\beta} A_{\alpha}=\left(\mathrm{d} A^{b}\right)_{\alpha \beta}, \\
\nabla_{\alpha} A^{\alpha}=\rho^{-1} \partial_{\alpha}\left(\rho A^{\alpha}\right)=-\delta A^{b},
\end{gathered}
$$

where $\rho$ is our Lorentzian density (2.12) (see Appendix A 1 for exterior calculus notation).

Proof of Lemma 4.5. Working in the neighborhood of a point $x$ and in chosen local coordinates, our diffeomorphism $\varphi$ can be written as

$$
\varphi^{\alpha}(x)=x^{\alpha}+A^{\alpha}(x)+O\left(|A|^{2}\right)
$$

[here, we made use of (4.1)] so that we have

$$
\frac{\partial \varphi^{\alpha}}{\partial x^{\beta}}=\delta_{\beta}^{\alpha}+\frac{\partial A^{\alpha}}{\partial x^{\beta}}+O\left(\left|A^{2}\right|\right)
$$

Solving (4.4) at first order in $A$ with initial condition (4.14) and using the fact that $\frac{d y^{\alpha}}{d s}=-A^{\alpha}+O\left(|A|^{2}\right)$, we get (4.10a).

Formula (4.10a) implies

$$
g^{\alpha \gamma} D^{\mu}{ }_{\gamma \mu \nu} D_{\beta}^{v}=\delta_{\beta}^{\alpha}+\nabla_{\beta} A^{\alpha}+\nabla^{\alpha} A_{\beta}+O\left(|A|^{2}\right),
$$

which gives us (4.10b) upon extracting the square root. 
Formula (4.10c) is now obtained by combining (4.10a) and (4.10b) with (4.7); in turn, it implies (4.10d) upon extracting the logarithm. Substituting (4.14) into (2.2) and, in turn, (2.2) into (2.3), we get (4.10e).

Finally, (4.10e) and the identity

$$
\operatorname{det} h_{\alpha \beta}=\operatorname{det} g_{\alpha \beta} \operatorname{det}(\mathrm{Id}+S)
$$

[cf. (2.3)] give us (4.10).

Remark 4.6. There is an alternative way of describing a diffeomorphism in terms of a vector field. This alternative approach is in the spirit of fluid mechanics and is based on Lie-algebraic considerations. Namely, consider a smooth vector field $u^{\alpha}(x)$, a field of "velocities," and the autonomous system of ordinary differential equations,

$$
\left\{\begin{array}{l}
\dot{y}=u(y), \\
\left.y\right|_{\tau=0}=x
\end{array}\right.
$$

that it generates. Here, $\tau \in[0,1]$ is a parameter and the dot stands for differentiation in $\tau$. We denote the solution of $(4.15)$ by $y(\tau ; x)$. For $u$ small enough, the map $x \mapsto y(1 ; x)$ realizes a diffeomorphism close to the identity. At a formal level, one would hope to generate an arbitrary diffeomorphism close to the identity by a suitable choice of vector field $u$. Furthermore, if we choose a divergence-free vector field, i.e., a vector field satisfying $\rho^{-1} \partial_{\alpha}\left(\rho u^{\alpha}\right)=0$ [compare with (4.10f) and (4.12)], then for $u$ small enough, the map $x \mapsto y(1 ; x)$ realizes a volumepreserving diffeomorphism close to the identity. Unfortunately, this approach does not work: it is known (Ref. 20, p. 163) that there does not exist a neighborhood of the identity where the exponential map exp : $\operatorname{Vect}(M) \rightarrow \operatorname{Diff}(M)$, from vector fields $u$ to diffeomorphisms, is surjective. There are simple explicit examples of diffeomorphisms of $\mathbb{S}^{1}$ arbitrarily close to the identity that cannot be represented in terms of the above flow (see, for example, Ref. 21, p. 1017; Ref. 22, pp. 8-9; and Ref. 23, pp. 456-457). The description of a diffeomorphism in terms of a vector field of displacements $A$ (see the beginning of this section) does not suffer from the deficiencies of the fluid mechanics description (4.15). The fundamental difference between the two approaches is that the concept of displacement relies on the use of the metric structure.

\section{LINEARIZED FIELD EQUATIONS}

Carrying on from Sec. IV, we assume that our diffeomorphism $\varphi: M \rightarrow M$ is sufficiently close to the identity map so that it can be described by a vector field of displacements $A(x)$. Furthermore, we can choose the local coordinates $y$ to be the same as $x$. Our aim in the current section is to linearize the field equations(3.21) and (2.11) in $A(x)$ and $p(x)$.

Formulas (4.10f) and (4.12) give us the linearization of the volume preservation condition (2.11),

$$
\delta A^{b}=0
$$

Formula (3.22) now reads

$$
(\mathrm{d} p)_{\alpha}(x)=\frac{\partial p}{\partial x^{\alpha}}(\varphi(x))
$$

and its linearization is the usual gradient,

$$
\frac{\partial p}{\partial x^{\alpha}}(x)
$$

The issue at hand is the linearization of $E(\varphi)$.

Inspection of formulas (2.5a)-(2.5d), (2.17b), and (4.10e) shows that the expansion of our Lagrangian $L\left(e_{2}(A), e_{3}(A), e_{4}(A)\right)$ in terms homogeneous in $A$ starts with the quadratic expression

$$
L^{(2)}(A)=-2\left(\nabla_{\alpha} A^{\alpha}\right)^{2}+\frac{1}{2}\left(\nabla_{\alpha} A_{\beta}+\nabla_{\beta} A_{\alpha}\right)\left(\nabla^{\alpha} A^{\beta}+\nabla^{\beta} A^{\alpha}\right)
$$

so that $L\left(e_{2}(A), e_{3}(A), e_{4}(A)\right)=L^{(2)}(A)+O\left(|A|^{3}\right)$. Variation of the quadratic action

$$
J^{(2)}(A)=\int_{M} L^{(2)}(A) \rho(x) d x
$$

generates the linearization $E^{(1)}(A)$ of $E(\varphi)$,

$$
\Delta J^{(2)}(A)=\int E_{\lambda}^{(1)}(A) \Delta A^{\lambda} \rho(x) d x
$$


However, prior to variation it is useful to rewrite (5.2) as in the following lemma whose proof is a straightforward computation.

Lemma 5.1. The Lagrangian (5.2) can be equivalently rewritten as

$$
L^{(2)}(A)=\frac{1}{2}\left(\nabla_{\alpha} A_{\beta}-\nabla_{\beta} A_{\alpha}\right)\left(\nabla^{\alpha} A^{\beta}-\nabla^{\beta} A^{\alpha}\right)-2 \operatorname{Ric}_{\mu \nu} A^{\mu} A^{v}+\nabla_{\kappa} B^{\kappa}
$$

where Ric is the Ricci tensor associated with $g$ and

$$
B^{\kappa}=-2\left[A^{\kappa}\left(\nabla_{\gamma} A^{\gamma}\right)-A^{\gamma}\left(\nabla_{\gamma} A^{\kappa}\right)\right]
$$

The divergence term $\nabla_{\kappa} B^{\kappa}$ in formula (5.3) does not contribute to the field equations, so we can replace our Lagrangian (5.2) with

$$
\tilde{L}^{(2)}(A)=\left\|\mathrm{d} A^{b}\right\|_{g}^{2}-2 \operatorname{Ric}(A, A)
$$

(see Appendix A 1 for exterior calculus notation). The advantage of writing our quadratic Lagrangian in the form (5.4) is that this representation does not involve covariant derivatives.

Formula (5.4) implies that the linearized operator generated by our action (2.15) reads

$$
E^{(1)}=2 \delta \mathrm{d}-4 \text { Ric. }
$$

In formulas (5.4) and (5.5), we abuse notation by using the symbol Ric for two different objects, the quadratic form on vectors Ric $(u, u)$ $:=\operatorname{Ric}_{\alpha \beta} u^{\alpha} u^{\beta}$ and the linear map on covectors Ric : $v_{\alpha} \mapsto \operatorname{Ric}_{\alpha \beta} v^{\beta}$.

Hence, our linearized field equations (3.21) and (2.11) read

$$
\left(\begin{array}{cc}
\delta \mathrm{d}-2 \text { Ric } & -\frac{1}{2} \mathrm{~d} \\
\delta & 0
\end{array}\right)\left(\begin{array}{c}
A^{b} \\
p
\end{array}\right)=0
$$

If we introduce a new scalar field

$$
\tilde{p}:=-\frac{1}{2} p
$$

the above system takes the form

$$
\left(\begin{array}{cc}
\delta \mathrm{d}-2 \mathrm{Ric} & \mathrm{d} \\
\delta & 0
\end{array}\right)\left(\begin{array}{c}
A^{b} \\
\tilde{p}
\end{array}\right)=0
$$

Let us now briefly discuss the analytic properties of the $5 \times 5$ matrix linear partial differential operator

$$
\text { Lin }: \Omega^{1}(M) \oplus \Omega^{0}(M) \rightarrow \Omega^{1}(M) \oplus \Omega^{0}(M), \quad\left(\begin{array}{l}
v \\
f
\end{array}\right) \mapsto\left(\begin{array}{cc}
\delta \mathrm{d}-2 \text { Ric } & \mathrm{d} \\
\delta & 0
\end{array}\right)\left(\begin{array}{l}
v \\
f
\end{array}\right)
$$

We start with the observation that the operator Lin is formally self-adjoint (symmetric) with respect to the $L^{2}$ inner product defined as in Appendix A 1.

The more specific properties of a linear differential operator are determined by its principal symbol. In local coordinates, the principal symbol is obtained by leaving only the leading (higher order) derivatives and replacing each partial differentiation $\partial / \partial x^{\alpha}$ by $i \xi_{\alpha}$, where $\xi$ is the dual variable (momentum) (see Ref. 24, Subsection 1.1.3). This gives a (matrix-)function on the cotangent bundle, the properties of which determine the basic features of the differential operator such as ellipticity or hyperbolicity. However, for our operator Lin matters are slightly more complicated because it has a block structure

$$
\left(\begin{array}{rr}
2^{\text {nd }} \text { order operator } & 1^{\text {st }} \text { order operator } \\
1^{\text {st }} \text { order operator } & 0 \text { order operator }
\end{array}\right)
$$

with operators of different orders in different blocks. Matrix operators with this particular structure are called Agmon-Douglis-Nirenberg type operators. ${ }^{25}$ Application of the Agmon-Douglis-Nirenberg construction gives the principal symbol of Lin as the linear map 


$$
\left(\begin{array}{l}
v \\
f
\end{array}\right) \mapsto\left(\begin{array}{c}
\|\xi\|_{g}^{2} v-\langle\xi, v\rangle_{g} \xi+i f \xi \\
-i\langle\xi, v\rangle_{g}
\end{array}\right)
$$

The determinant of the linear map (5.9) is

$$
-\|\xi\|_{g}^{8} .
$$

Now, if our metric $g$ were Riemannian, then the quantity (5.10) would not vanish on $T^{*} M \backslash\{0\}$, and hence, our operator Lin would be elliptic in the Agmon-Douglis-Nirenberg sense. However, for Lorentzian metric $g$, the quantity (5.10) vanishes on light cones, which suggests that our operator Lin is hyperbolic. There is an extensive literature dealing with Agmon-Douglis-Nirenberg type operators in the elliptic setting, but we are unaware of similar results for the hyperbolic case. A rigorous investigation of well-posedness issues for the operator Lin is, though, outside the scope of our paper. For a review of different notions of hyperbolicity in a setting similar to ours, see Ref. 26, Sec. 4 .

Note that if we replace the $5 \times 5$ matrix operator (5.8) with the $4 \times 4$ matrix operator $\delta \mathrm{d}$, then the principal symbol will be a degenerate matrix whose determinant is identically zero.

Let us now assume that our spacetime $(M, g)$ is Ricci-flat,

$$
\text { Ric }=0 .
$$

Note that condition (5.11) is the accepted relativistic definition of vacuum. Moreover, it is easy to see that if $(M, g)$ is Ricci-flat, then so is $(M, h)$.

Under condition (5.11), Eq. (5.7) implies

$$
\delta \mathrm{d} \tilde{p}=\square_{g} \tilde{p}=0 .
$$

We see that we have a separate equation for the scalar field $\tilde{p}$, the wave equation. This observation allows us to collect solutions of our system (5.7) into equivalence classes corresponding to particular choices of $\tilde{p}$ : we say that two solutions, $\left(\begin{array}{c}A^{b} \\ \tilde{p}\end{array}\right)$ and $\left(\begin{array}{c}A^{b^{\prime}} \\ \tilde{p}^{\prime}\end{array}\right)$, are equivalent if $\tilde{p}=\tilde{p}^{\prime}$.

Let us now fix a particular solution $\tilde{p}$ of the wave equation and work within the corresponding equivalence class. Then, the first four equations from our system (5.7) can be rewritten as

$$
\delta \mathrm{d} A^{b}=J,
$$

where $J:=-\mathrm{d} \tilde{p}$. We have arrived at Maxwell's equations in the Lorenz gauge (5.1) and with exact current $J \in \mathrm{d} \Omega^{0}(M)$. Recovering Maxwell's equations in the Lorenz gauge is not a factitious artifact of our theory, but, in a sense, a natural thing to have: this is what one obtains when looking at irreducible representations of the Poincaré group in the spirit of Wigner's classification (cf. Ref. 27, Chap. 21).

\section{HOMOGENEOUS DIFFEOMORPHISMS}

In the remainder of this paper, we will construct explicit solutions of the nonlinear field equation (3.21). Specifically, we will write down explicitly volume preserving diffeomorphisms $\varphi$ satisfying (3.21) with $p=0$. In other words, we will present volume preserving solutions of the unconstrained nonlinear field equation (3.5).

Seeking such solutions constitutes an overdetermined problem: we are looking at a system of five nonlinear partial differential equations (3.5) and (2.11) for four unknowns, the functions $\varphi^{\alpha}(x), \alpha=1,2,3,4$, appearing in the local representation (2.1) of our diffeomorphism $\varphi$. We will base our construction on group-theoretic ideas, the essence of which is explained below.

Further on, $\operatorname{Isom}(M, g)$ denotes the finite-dimensional subgroup of $\operatorname{Diff}(M)$ comprising diffeomorphisms that are isometries.

Definition 6.1. Let $\varphi \in \operatorname{Diff}(M)$. We say that $\varphi$ is homogeneous if there exists a subgroup $H \subset \operatorname{Isom}(M, g)$ acting transitively on $M$ and satisfying

$$
H \circ \varphi=\varphi \circ H .
$$

If we have the stronger property

$$
\xi \circ \varphi=\varphi \circ \xi, \quad \forall \xi \in H,
$$

we say that $\varphi$ is equivariant.

In other words, condition (6.1) can be rewritten as follows: for any $\xi \in H$, there exists a $\eta \in H$ such that the diagram

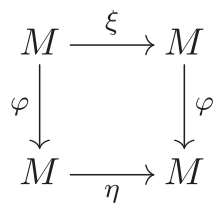

is commutative. 
Theorem 6.2. Let $\varphi$ be a homogeneous diffeomorphism. Then, the scalar invariants (2.5) are constant. Furthermore, if the covector field $E(\varphi)$ defined in accordance with formula (3.4) vanishes at a point then it vanishes identically.

Proof. Let us prove the second statement first. Let $\varphi$ be a homogeneous diffeomorphism and $x, y \in M$ two arbitrary points. We will assume that $\left.E(\varphi)\right|_{x}=0$, and we will show that $\left.E(\varphi)\right|_{y}=0$. In view of Definition 6.1, there exist isometries $\xi$ and $\eta$ such that

$$
y=\xi(x), \quad \varphi(y)=\eta(\varphi(x))
$$

and

$$
\eta \circ \varphi=\varphi \circ \xi
$$

Note that in writing (6.3), we only used the transitivity of the action of $H$ on $M$, whereas (6.4) required the use of the additional condition (6.1).

It is possible to choose coordinates in some neighborhoods $\mathcal{U}(x)$ and $\mathcal{U}(\varphi(x))$ of $x$ and $\varphi(x)$, respectively, in such a way that $\varphi$ is locally the identity map,

$$
\left.\varphi\right|_{\mathcal{U}(x)} \simeq \operatorname{id}: \mathcal{U}(x) \rightarrow \mathcal{U}(\varphi(x))
$$

We can then prescribe coordinates in some neighborhood $\mathcal{U}(y)$ of $y$ [respectively, $\mathcal{U}(\varphi(y))$ of $\varphi(y)$ ] via the isometry $\xi$ (respectively, $\eta$ ). This has two consequences. First, the map

$$
\left.\varphi\right|_{\mathcal{U}(y)}: \mathcal{U}(y) \rightarrow \mathcal{U}(\varphi(y))
$$

is the identity in our local coordinates. Second, in this coordinate representation, the components of the metric tensor are the same near $x$ and $y$ and near $\varphi(x)$ and $\varphi(y)$. This can be easily seen by explicitly imposing the isometry conditions $\xi^{*} g=g$ and $\eta^{*} g=g$ locally, after observing that $\left.\xi\right|_{\mathcal{U}(x)} \simeq \mathrm{id}$ and $\left.\eta\right|_{\mathcal{U}(\varphi(x))} \simeq$ id for our choice of coordinates. In particular, the Jacobian of the change of coordinates from coordinates centered at $x$ [respectively, $\varphi(x)$ ] to coordinates centered at $y$ [respectively, $\varphi(y)]$ is 1 . The local expression (3.4) of $E(\varphi)$ depends only on the (local representation of the) metric, $\varphi$, and its derivatives. Since such local representations are the same in neighborhoods of $x$ and $y,\left.E(\varphi)\right|_{x}=0$ implies $\left.E(\varphi)\right|_{y}=0$.

Finally, let us prove that the scalar invariants are constant. If we compute the scalar invariants in local coordinates, we realize that they only depend on the local representation of the metric, of $\varphi$ and of its first derivatives [see (2.3) and (2.5)]. Since such representations can be made the same in the neighborhood of any pair of points $x$ and $y$, as described above, it ensures that the scalar invariants take the same value everywhere, namely, they are constant.

Theorem 6.2 tells us that if we seek a solution of nonlinear field equation(3.5) in the form of a homogeneous diffeomorphism, then it is sufficient to satisfy these field equations at a single point.

Remark 6.3. Note that our mathematical model is invariant under the action of the group of isometries in the following sense. Let $\varphi \in \operatorname{Diff}_{\rho}(M)$ and $p: M \rightarrow \mathbb{R}$ be a solution of our field equation(3.21), and let $\xi \in \operatorname{Isom}(M, g)$ be an arbitrary isometry. Then, $\xi \circ \varphi$ and $p \circ \xi^{-1}$ is also a solution.

\section{SPECIAL SUBGROUPS OF THE POINCARÉ GROUP}

In the remainder of this paper, we work in Minkowski space $\mathbb{M}$ where the metric is $g_{\alpha \beta}=\operatorname{diag}(1,1,1,-1)$. Further on, Poinc $(\mathbb{M})$ $:=\operatorname{Isom}\left(\mathbb{R}^{4}, g\right)$ denotes the ten-dimensional group of isometries of $\mathbb{M}$, commonly known as the Poincaré group. Clearly, Poinc $(\mathbb{M})=\mathbb{R}^{4}$ $\rtimes \mathrm{O}(3,1)$.

In fact, we will be working with the identity component of the Poincaré group, $\operatorname{ISO}^{+}(3,1)$. This is known to be the fundamental symmetry group of physics in that it turns inertial frames into one another.

The Poincaré group can be realized as a subgroup of the matrix group $\operatorname{SL}(5, \mathbb{R})$ as follows:

$$
\mathbb{R}^{4} \rtimes \mathrm{O}(3,1) \ni(v, \Lambda) \mapsto\left(\begin{array}{ccccc} 
& & & v \\
0 & 0 & 0 & 0 & 1
\end{array}\right) \in \mathrm{SL}(5, \mathbb{R}) .
$$

Here, the $5 \times 5$ matrix acts on $x \in \mathbb{M}$ by matrix vector multiplication after complementing it with 1 ,

$$
\left(\begin{array}{l}
x \\
1
\end{array}\right) \mapsto\left(\begin{array}{ccccc} 
& & & v \\
0 & 0 & 0 & 0 & 1
\end{array}\right)\left(\begin{array}{l}
x \\
1
\end{array}\right) .
$$


We now introduce special subgroups of the restricted Poincaré group $\operatorname{ISO}^{+}(3,1)$, which will be used in Secs. VIII and IX.

Definition 7.1. The right-handed massless screw group $\mathrm{SG}_{0}^{+}$and left-handed massless screw group $\mathrm{SG}_{0}^{-}$are the subgroups of $\operatorname{ISO}^{+}(3,1)$ realized in matrix representation by

$$
\mathrm{SG}_{0}^{ \pm}:=\left\{\left(\begin{array}{ccccc}
\cos \left(q^{3}+q^{4}\right) & \mp \sin \left(q^{3}+q^{4}\right) & 0 & 0 & q^{1} \\
\pm \sin \left(q^{3}+q^{4}\right) & \cos \left(q^{3}+q^{4}\right) & 0 & 0 & q^{2} \\
0 & 0 & 1 & 0 & q^{3} \\
0 & 0 & 0 & 1 & q^{4} \\
0 & 0 & 0 & 0 & 1
\end{array}\right) \mid q \in \mathbb{R}^{4}\right\} .
$$

Definition 7.2. Let $m$ be a positive real number. The massive screw group $\mathrm{SG}_{m}$ is the subgroup of $\operatorname{ISO}^{+}(3,1)$ realized in matrix representation by

$$
\mathrm{SG}_{m}:=\left\{\left(\begin{array}{ccccc}
\cos \left(2 m q^{4}\right) & -\sin \left(2 m q^{4}\right) & 0 & 0 & q^{1} \\
\sin \left(2 m q^{4}\right) & \cos \left(2 m q^{4}\right) & 0 & 0 & q^{2} \\
0 & 0 & 1 & 0 & q^{3} \\
0 & 0 & 0 & 1 & q^{4} \\
0 & 0 & 0 & 0 & 1
\end{array}\right) \mid q \in \mathbb{R}^{4}\right\} .
$$

It is easy to see that $\mathrm{SG}_{0}^{+}, \mathrm{SG}_{0}^{-}$, and $\mathrm{SG}_{m}$ are indeed subgroups of $\mathrm{ISO}^{+}(3,1)$ and act transitively on $\mathrm{M}$. Each of these groups is isomorphic to the direct product of $\mathbb{R}$ with a three-dimensional group of Bianchi type $\mathrm{Bi}\left(\mathrm{VII}_{0}\right)$.

Let $\xi \in \operatorname{ISO}^{+}(3,1)$. Then, $\xi^{-1} \mathrm{SG}_{0}^{+} \xi, \xi^{-1} \mathrm{SG}_{0}^{-} \xi$, and $\xi^{-1} \mathrm{SG}_{m} \xi$ are also subgroups of $\mathrm{ISO}^{+}(3,1)$. The question we want to address is what happens under conjugation.

Lemma 7.3. There does not exist a $\xi \in \mathrm{ISO}^{+}(3,1)$ such that $\xi^{-1} \mathrm{SG}_{0}^{+} \xi=\mathrm{SG}_{0}^{-}$.

Proof. The result follows from Lemma C.1: the Hodge dual of axial torsion associated with the two groups lies on opposite sides of the light cone and conjugation by an element of $\operatorname{ISO}^{+}(3,1)$ cannot change this.

Lemma 7.3 tells us that the groups $\mathrm{SG}_{0}^{+}$and $\mathrm{SG}_{0}^{-}$are genuinely different in that one cannot be turned into the other by conjugation.

Let us now examine what happens when we conjugate the massive screw group. It turns out that the situation here is completely different. Specifically, choose $\xi=\operatorname{diag}(-1,-1,-1,-1,1)$ to be the PT transformation. Then,

$$
\begin{aligned}
\xi^{-1} \mathrm{SG}_{m} \xi & =\left\{\left(\begin{array}{ccccc}
\cos \left(2 m q^{4}\right) & -\sin \left(2 m q^{4}\right) & 0 & 0 & -q^{1} \\
\sin \left(2 m q^{4}\right) & \cos \left(2 m q^{4}\right) & 0 & 0 & -q^{2} \\
0 & 0 & 1 & 0 & -q^{3} \\
0 & 0 & 0 & 1 & -q^{4} \\
0 & 0 & 0 & 0 & 1
\end{array}\right) \mid q \in \mathbb{R}^{4}\right\} \\
& =\left\{\left(\begin{array}{ccccc}
\cos \left(2 m q^{4}\right) & \sin \left(2 m q^{4}\right) & 0 & 0 & q^{1} \\
-\sin \left(2 m q^{4}\right) & \cos \left(2 m q^{4}\right) & 0 & 0 & q^{2} \\
0 & 0 & 1 & 0 & q^{3} \\
0 & 0 & 0 & 1 & q^{4} \\
0 & 0 & 0 & 0 & 1
\end{array}\right) \mid q \in \mathbb{R}^{4}\right\} .
\end{aligned}
$$

This means that a different choice of signs in (7.2) does not yield a different family of subgroups. The argument presented in this paragraph is in agreement with Lemma C.1: the Hodge dual of axial torsion associated with the massive group is spacelike and conjugation moves this covector without encountering obstructions.

\section{EXPLICIT MASSLESS SOLUTIONS OF NONLINEAR FIELD EQUATIONS}

Working in Minkowski space $\mathbb{M}$, we will describe our diffeomorphism $\varphi$ by a vector field of displacements,

$$
\varphi: x^{\alpha} \mapsto x^{\alpha}+A^{\alpha}(x) .
$$

The concept of a vector field of displacements was introduced in Sec. IV. The special feature of Minkowski space is that we do not need to assume that our diffeomorphism is sufficiently close to the identity map. The only restriction on the choice of vector field $A$ is

$$
\operatorname{det}\left(D^{\alpha}{ }_{\beta}\right) \neq 0 \text {, }
$$


where

$$
D_{\beta}^{\alpha}=\delta_{\beta}^{\alpha}+\partial A^{\alpha} / \partial x^{\beta}
$$

is the deformation gradient [see formula (4.5) and associated discussion]. Condition (8.2) ensures that we do indeed have a diffeomorphism, a smooth invertible map.

We seek volume preserving solutions. Examination of formula (4.6) shows that in Minkowski space, the volume preservation condition (2.11) reduces to $\left|\operatorname{det}\left(D^{\alpha}{ }_{\beta}\right)\right|=1$, which means that we either have

$$
\operatorname{det}\left(D_{\beta}^{\alpha}\right)=+1
$$

or

$$
\operatorname{det}\left(D_{\beta}^{\alpha}\right)=-1 \text {. }
$$

Solutions presented in this section and Sec. IX will possess the property (8.4a).

We say that a real lightlike covector $p=\left(p_{1}, p_{2}, p_{3}, p_{4}\right)$ lies on the forward light cone if $p_{4}>0$. We say that a complex vector $u=\left(u^{1}, u^{2}, u^{3}, u^{4}\right)$ is isotropic if $u_{\alpha} \bar{u}^{\alpha}>0$ and $u_{\alpha} u^{\alpha}=0$.

The use of the term "isotropic" is motivated by Cartan who used it in the three-dimensional Euclidean setting. If we choose a coordinate system such that $u^{4}=0$, our definition is equivalent to that in Ref. 28 (Chap. III, Sec. I).

Theorem 8.1. Let $p$ be a real lightlike covector on the forward light cone, let $u$ be a complex isotropic vector orthogonal to $p$, and let

$$
\mathbb{A}^{\alpha}(x)=u^{\alpha} e^{i p_{\beta} x^{\beta}} .
$$

Then, the diffeomorphism (8.1) with

$$
A(x)=\operatorname{Re}[\mathbb{A}(x)]
$$

is volume preserving and satisfies the nonlinear field equations (3.5).

Proof. We can perform a (unique) proper orthochronous Lorentz transformation of coordinates so that formula (8.5) reads

$$
\mathbb{A}^{\alpha}(x)=a\left(\begin{array}{c}
1 \\
\mp i \\
0 \\
0
\end{array}\right) e^{i\left(x^{3}+x^{4}\right)},
$$

where $a=\sqrt{u_{\alpha} \bar{u}^{\alpha} / 2}$. Then, (8.6) becomes

$$
A^{\alpha}(x)=a\left(\begin{array}{c}
\cos \left(x^{3}+x^{4}\right) \\
\pm \sin \left(x^{3}+x^{4}\right) \\
0 \\
0
\end{array}\right) .
$$

Substituting (8.8) into (8.3), we get the following explicit formula for the deformation gradient:

$$
D_{\beta}^{\alpha}=\left(\begin{array}{cccc}
1 & 0 & -a \sin \left(x^{3}+x^{4}\right) & -a \sin \left(x^{3}+x^{4}\right) \\
0 & 1 & \pm a \cos \left(x^{3}+x^{4}\right) & \pm a \cos \left(x^{3}+x^{4}\right) \\
0 & 0 & 1 & 0 \\
0 & 0 & 0 & 1
\end{array}\right),
$$

where the first tensor index, $\alpha$, enumerates the rows and the second, $\beta$, the columns. It is immediately clear that (8.4a) is satisfied. Substituting now (8.9) into (4.6) and (2.3), we get the following explicit formula for the strain tensor:

$$
S_{\beta}^{\alpha}=\left(\begin{array}{cccc}
0 & 0 & -a \sin \left(x^{3}+x^{4}\right) & -a \sin \left(x^{3}+x^{4}\right) \\
0 & 0 & \pm a \cos \left(x^{3}+x^{4}\right) & \pm a \cos \left(x^{3}+x^{4}\right) \\
-a \sin \left(x^{3}+x^{4}\right) & \pm a \cos \left(x^{3}+x^{4}\right) & a^{2} & a^{2} \\
a \sin \left(x^{3}+x^{4}\right) & \mp a \cos \left(x^{3}+x^{4}\right) & -a^{2} & -a^{2}
\end{array}\right) .
$$

It is easy to check that the matrix (8.10) is nilpotent, so all our scalar invariants (2.5) vanish identically. Note that the nilpotency index of (8.10) is three, which, according to Lemma B.1, is the maximal possible. 
We vary the vector field of displacements $A(x)$ as

$$
A^{\alpha}(x) \mapsto A^{\alpha}(x)+\Delta A^{\alpha}(x)
$$

This generates an increment of our scalar invariants $\Delta e_{j}$ and an increment of our Lagrangian

$$
\left.\sum_{j=2}^{4} \frac{\partial L}{\partial e_{j}}\right|_{e_{2}=e_{3}=e_{4}=0} \Delta e_{j} .
$$

In order to prove that our diffeomorphism satisfies the nonlinear field equation (3.5), it is sufficient to prove that

$$
\int_{\mathbb{R}^{4}} \Delta e_{j} d x=0, \quad j=2,3,4
$$

Straightforward calculations give

$$
\begin{aligned}
& \Delta e_{1}=2\left(\delta^{\beta}{ }_{\alpha}+\frac{\partial A_{\alpha}}{\partial x_{\beta}}\right) \frac{\partial \Delta A^{\alpha}}{\partial x^{\beta}}, \\
& \Delta e_{2}=-2\left(a^{2} p^{\beta} p_{\alpha}+\frac{\partial A^{\beta}}{\partial x^{\alpha}}+\frac{\partial A_{\alpha}}{\partial x_{\beta}}\right)\left(\frac{\partial \Delta A^{\alpha}}{\partial x^{\beta}}+\frac{\partial A_{\gamma}}{\partial x_{\alpha}} \frac{\partial \Delta A^{\gamma}}{\partial x^{\beta}}\right), \\
& \Delta e_{3}=2 a^{2} p^{\beta} p_{\alpha}\left(\frac{\partial \Delta A^{\alpha}}{\partial x^{\beta}}+\frac{\partial A_{\gamma}}{\partial x_{\alpha}} \frac{\partial \Delta A^{\gamma}}{\partial x^{\beta}}\right)=2 a^{2} p^{\beta} p_{\alpha} \frac{\partial \Delta A^{\alpha}}{\partial x^{\beta}}, \\
& \Delta e_{4}=0
\end{aligned}
$$

where $p_{\kappa}=(0,0,1,1)$. Integrating $(8.12)-(8.12 \mathrm{~d})$ by parts and using the identities

$$
\square A=0, \quad \frac{\partial A^{\alpha}}{\partial x^{\alpha}}=0, \quad\left(p^{\alpha} \frac{\partial}{\partial x^{\alpha}}\right) A=0,
$$

we arrive at (8.11).

The crucial element of the above proof is the observation that the scalar invariants (2.5) generated by the diffeomorphism (8.1) and (8.8) are constant. We established this fact by means of explicit analytic calculations. However, at a group-theoretic level, this follows from Theorem 6.2. Indeed, take an arbitrary $\xi \in \mathrm{SG}_{0}^{ \pm}$[see formula (7.1)]. This isometry acts as

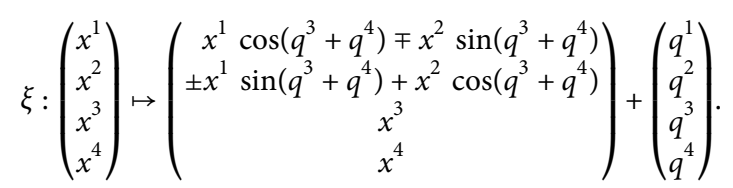

Our diffeomorphism (8.1) and (8.8) acts as

$$
\varphi_{ \pm}:\left(\begin{array}{l}
x^{1} \\
x^{2} \\
x^{3} \\
x^{4}
\end{array}\right) \mapsto\left(\begin{array}{l}
x^{1} \\
x^{2} \\
x^{3} \\
x^{4}
\end{array}\right)+a\left(\begin{array}{c}
\cos \left(x^{3}+x^{4}\right) \\
\pm \sin \left(x^{3}+x^{4}\right) \\
0 \\
0
\end{array}\right)
$$

and its inverse acts as

$$
\varphi_{ \pm}^{-1}:\left(\begin{array}{c}
x^{1} \\
x^{2} \\
x^{3} \\
x^{4}
\end{array}\right) \mapsto\left(\begin{array}{l}
x^{1} \\
x^{2} \\
x^{3} \\
x^{4}
\end{array}\right)-a\left(\begin{array}{c}
\cos \left(x^{3}+x^{4}\right) \\
\pm \sin \left(x^{3}+x^{4}\right) \\
0 \\
0
\end{array}\right)
$$

Composing $\xi$ with $\varphi_{ \pm}$, we get 


$$
\begin{array}{r}
\xi \circ \varphi_{ \pm}:\left(\begin{array}{c}
x^{1} \\
x^{2} \\
x^{3} \\
x^{4}
\end{array}\right) \mapsto\left(\begin{array}{c}
x^{1} \cos \left(q^{3}+q^{4}\right) \mp x^{2} \sin \left(q^{3}+q^{4}\right) \\
\pm x^{1} \sin \left(q^{3}+q^{4}\right)+x^{2} \cos \left(q^{3}+q^{4}\right) \\
x^{3} \\
x^{4}
\end{array}\right)+\left(\begin{array}{c}
q^{1} \\
q^{2} \\
q^{3} \\
q^{4}
\end{array}\right) \\
+a\left(\begin{array}{c}
\cos \left(x^{3}+q^{3}+x^{4}+q^{4}\right) \\
\pm \sin \left(x^{3}+q^{3}+x^{4}+q^{4}\right) \\
0 \\
0
\end{array}\right) .
\end{array}
$$

Finally, a composition with $\varphi_{ \pm}^{-1}$ gives us

$$
\begin{aligned}
\varphi_{ \pm}^{-1} \circ \xi \circ \varphi_{ \pm}:\left(\begin{array}{c}
x^{1} \\
x^{2} \\
x^{3} \\
x^{4}
\end{array}\right) & \mapsto\left(\begin{array}{c}
x^{1} \cos \left(q^{3}+q^{4}\right) \mp x^{2} \sin \left(q^{3}+q^{4}\right) \\
\pm x^{1} \sin \left(q^{3}+q^{4}\right)+x^{2} \cos \left(q^{3}+q^{4}\right) \\
x^{3} \\
x^{4}
\end{array}\right)+\left(\begin{array}{c}
q^{1} \\
q^{2} \\
q^{3} \\
q^{4}
\end{array}\right) \\
& +a\left(\begin{array}{c}
\cos \left(x^{3}+q^{3}+x^{4}+q^{4}\right) \\
\pm \sin \left(x^{3}+q^{3}+x^{4}+q^{4}\right) \\
0 \\
0
\end{array}\right)-a\left(\begin{array}{c}
\cos \left(x^{3}+q^{3}+x^{4}+q^{4}\right) \\
\pm \sin \left(x^{3}+q^{3}+x^{4}+q^{4}\right) \\
0 \\
0
\end{array}\right),
\end{aligned}
$$

which means that $\varphi_{ \pm}^{-1} \circ \xi \circ \varphi_{ \pm}=\xi$. Thus, our diffeomorphism $\varphi_{ \pm}$is equivariant as per Definition 6.1 with $H=\mathrm{SG}_{0}^{ \pm}$.

Observe now that the complex 2 -form $p \wedge u^{b}$ is an eigenvector of the Hodge star. This motivates the following definition:

Definition 8.2. We say that a solution from Theorem 8.1 is right-handed if $*\left(p \wedge u^{b}\right)=i\left(p \wedge u^{b}\right)$ and left-handed if $*\left(p \wedge u^{b}\right)=-i\left(p \wedge u^{b}\right)$.

It is easy to see that the upper sign in formula (8.8) corresponds to a right-handed solution and the lower sign corresponds to a lefthanded one. Note that we defined right-/left-handedness for groups (Definition 7.1) and massless solutions (Definition 8.2) in such a way that they agree.

\section{EXPLICIT MASSIVE SOLUTIONS OF NONLINEAR FIELD EQUATIONS}

Theorem 9.1. Let $m$ be a positive real number, and let $p$ be a real timelike covector with $p_{\beta} p^{\beta}=-4 m^{2}$ and $p_{4}>0$. Let $u$ be a complex isotropic vector orthogonal to $p$, and let $v$ be a real vector orthogonal to $p$ and $u$. Suppose that

$$
4 m^{2}\left(\frac{1}{2} u_{\alpha} \bar{u}^{\alpha}+v_{\beta} v^{\beta}\right)=c
$$

where $c$ is a critical point from (2.18), and put

$$
\mathbb{A}^{\alpha}(x)=u^{\alpha} e^{i p_{\beta} x^{\beta}} .
$$

Then, the diffeomorphism (8.1) with

$$
A(x)=\operatorname{Re}[\mathbb{A}(x)]+\left(p_{\gamma} x^{\gamma}\right) v
$$

is volume preserving and satisfies the nonlinear field equations (3.5).

Remark 9.2. It is easy to see that under the assumptions of Theorem 9.1, the scalar $\left\|\mathrm{d} A^{b}\right\|_{g}^{2}$ is constant,

$$
\left\|\mathrm{d} A^{b}\right\|_{g}^{2}=-4 m^{2}\left(\frac{1}{2} u_{\alpha} \bar{u}^{\alpha}+v_{\beta} v^{\beta}\right) .
$$

Hence, formula (9.1) can be equivalently rewritten as

$$
\left\|\mathrm{d} A^{b}\right\|_{g}^{2}=-c,
$$

which is a condition on the strength of the field $\mathrm{d} A^{\text {b }}$. We see a certain similarity with the Born-Infeld model ${ }^{29}$ (Ref. 30, Sec. 2.1), which sets constraints on the admissible values of $\left\|\mathrm{d} A^{b}\right\|_{g}^{2}$. 
Proof of Theorem 9.1. Arguing as in the Proof of Theorem 8.1, we can perform a (unique) proper orthochronous Lorentz transformation of coordinates so that formula $(9.2)$ reads

$$
\mathbb{A}^{\alpha}(x)=a\left(\begin{array}{c}
1 \\
-i \\
0 \\
0
\end{array}\right) e^{2 i m x^{4}}
$$

and (9.3) becomes

$$
A^{\alpha}(x)=\left(\begin{array}{c}
a \cos \left(2 m x^{4}\right) \\
a \sin \left(2 m x^{4}\right) \\
2 m b x^{4} \\
0
\end{array}\right)
$$

Here,

$$
\begin{aligned}
& a=\sqrt{\frac{u_{\alpha} \bar{u}^{\alpha}}{2}}, \\
& b=-\frac{i}{4 m a^{2}} *\left(p \wedge u^{b} \wedge \bar{u}^{b} \wedge v^{b}\right) .
\end{aligned}
$$

Note that $|b|=\sqrt{v_{\alpha} v^{\alpha}}$. However, in defining the scalar invariant $b$, we used the seemingly more complicated formula (9.7b) in order to capture information on the relative orientation of the four covectors $p, \operatorname{Re} u^{b}, \operatorname{Im} u^{b}$, and $v^{b}$. With this notation, formula (9.1) can be rewritten as

$$
4 m^{2}\left(a^{2}+b^{2}\right)=c .
$$

The corresponding deformation gradient reads

$$
D_{\beta}^{\alpha}=\left(\begin{array}{cccc}
1 & 0 & 0 & -2 m a \sin \left(2 m x^{4}\right) \\
0 & 1 & 0 & 2 m a \cos \left(2 m x^{4}\right) \\
0 & 0 & 1 & 2 m b \\
0 & 0 & 0 & 1
\end{array}\right)
$$

for which (8.4a) is satisfied. The resulting strain tensor is

$$
S_{\beta}^{\alpha}=\left(\begin{array}{cccc}
0 & 0 & 0 & -2 m a \sin \left(2 m x^{4}\right) \\
0 & 0 & 0 & 2 m a \cos \left(2 m x^{4}\right) \\
0 & 0 & 0 & 2 m b \\
2 m a \sin \left(2 m x^{4}\right) & -2 m a \cos \left(2 m x^{4}\right) & -2 m b & -c
\end{array}\right) .
$$

Unlike (8.10), the matrix (9.9) is not nilpotent: its eigenvalues are zero (algebraic and geometric multiplicity two) and

$$
-\frac{c}{2} \pm \frac{\sqrt{c(c-4)}}{2}
$$

The matrix is diagonalizable if and only if $c \neq 4$.

The fact that the eigenvalues of the strain tensor (9.9) are constant implies that all our scalar invariants (2.5) are constant,

$$
e_{1}=-c, \quad e_{2}=c, \quad e_{3}=e_{4}=0
$$

Arguing as in the Proof of Theorem 8.1, we see that in order to prove that our diffeomorphism satisfies the nonlinear field equation (3.5), it is sufficient to show, in view of (2.18), that

$$
\int_{\mathbb{R}^{4}} \Delta e_{j} d x=0, \quad j=3,4
$$

It is easy to see that $\Delta e_{4}=0$, which, in essence, is to do with the fact that zero is a double eigenvalue of (9.9). 


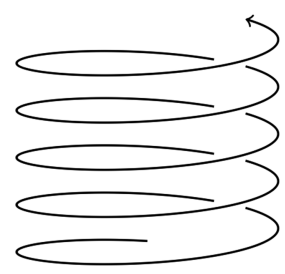

(i) $b>0$

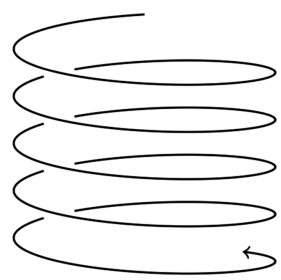

(ii) $b<0$

FIG. 1. Massive solution.

The formula for $\Delta e_{3}$ reads

$$
\Delta e_{3}=B_{\alpha}^{\beta} \frac{\partial \Delta A^{\alpha}}{\partial x^{\beta}}
$$

where $B^{\beta}{ }_{\alpha}$ is some tensor. The explicit formulas for the components of this tensor are complicated; however, for our purposes, it suffices to observe that $B^{4}{ }_{\alpha}=0$ and that the remaining components depend only on the coordinate $x^{4}$. Hence, integration by parts yields

$$
\int_{\mathbb{R}^{4}} \Delta e_{3} d x=-\int_{\mathbb{R}^{4}}\left(\frac{\partial B^{\beta}{ }_{\alpha}}{\partial x^{\beta}}\right) \Delta A^{\alpha} d x=-\int_{\mathbb{R}^{4}}\left(\frac{\partial B^{4}{ }_{\alpha}}{\partial x^{4}}\right) \Delta A^{\alpha} d x=0 .
$$

Group-theoretic arguments apply to the massive case as well. Taking an arbitrary $\xi \in \mathrm{SG}_{m}$ [see formula $(7.2)$ ], we get $\varphi^{-1} \circ \xi \circ \varphi=\eta$, where

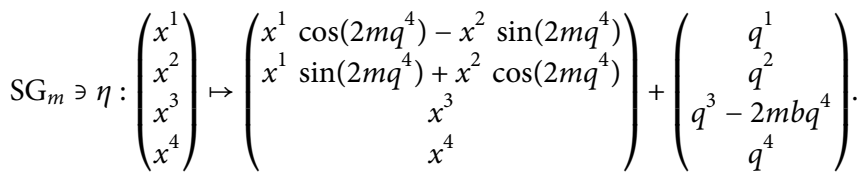

This means that our diffeomorphism $\varphi$ is homogeneous as per Definition 6.1 with $H=\mathrm{SG}_{m}$. It is equivariant if and only if $b=0$.

Let us discuss the continuum mechanics interpretation of formula (9.6). We are looking at a translation (rigid motion without rotation) of three-dimensional Euclidean space, which is a function of the time coordinate $x^{4}$. Every point of three-dimensional Euclidean space moves along a helix, [see Fig. 1(i) for $b>0$ and Fig. 1(ii) for $b<0$ ].

The parameter $b$ could be interpreted as electric charge. Note that for the given values of positive parameters $m$ and $a$, the parameter $b$ can take only two values,

$$
b= \pm \sqrt{\frac{c}{4 m^{2}}-a^{2}}
$$

\section{MASSLESS DIRAC EQUATION}

Let the diffeomorphisms $\varphi_{+}$and $\varphi_{-}$be right-handed and left-handed massless solutions as per Definition 8.2. In this section, we will calculate the corresponding rotation 2 -forms (see Sec. IV) and show that they are equivalent to spinor fields which satisfy massless Dirac equations.

The deformation gradient reads

$$
D_{\beta}^{\alpha}=\delta_{\beta}^{\alpha}+\operatorname{Re}\left[i u^{\alpha} p_{\beta} e^{i p_{\gamma} x^{\gamma}}\right] .
$$

In a particular coordinate system, the above formula turns to (8.9). Performing a polar decomposition (4.7), we get

$$
U_{\beta}^{\alpha}=\delta_{\beta}^{\alpha}-\frac{1}{2} \operatorname{Re}\left[i\left(p^{\alpha} u_{\beta}-u^{\alpha} p_{\beta}\right) e^{i p_{\gamma} x^{\gamma}}\right]-\frac{u_{\gamma} \bar{u}^{\gamma}}{16} p^{\alpha} p_{\beta} \text {, }
$$




$$
V_{\beta}^{\alpha}=\delta_{\beta}^{\alpha}+\frac{1}{2} \operatorname{Re}\left[i\left(p^{\alpha} u_{\beta}+u^{\alpha} p_{\beta}\right) e^{i p_{\gamma} x^{\gamma}}\right]+\frac{3 u_{\gamma} \bar{u}^{\gamma}}{16} p^{\alpha} p_{\beta}
$$

On account of formula (4.8), one can compute the logarithm of (10.2), lower the first index, and obtain the following explicit formula for the rotation 2-form:

$$
F=-\frac{1}{2} \operatorname{Re}\left[i\left(p \wedge u^{b}\right) e^{i p_{\gamma} x^{y}}\right]=-\frac{1}{2} \mathrm{~d} A^{b}
$$

We see that the formula for our rotation 2 -form is remarkably simple. Recall that for a general diffeomorphism, we have $F=-\frac{1}{2} \mathrm{~d} A^{b}$ $+O\left(|A|^{2}\right)$ [see formulas (4.10d) and (4.11)]. However, the deformation gradient generated by our massless solutions is very special and turns out to be linear in displacements, without any second (or higher) order terms and without any assumptions on the amplitude. The underlying reason for such simplicity is that at any given point of $\mathbb{M}$, one can identify a two-dimensional invariant subspace of the tangent fiber in which the deformation gradient (10.1) differs from the identity map. Furthermore, the restriction of the Minkowski metric to this subspace is degenerate.

Put

$$
\mathbb{F}:=-\frac{1}{2} \mathrm{~d} \mathbb{A}^{b}=-\frac{i}{2}\left(p \wedge u^{b}\right) e^{i p_{y} x^{y}}
$$

so that $F=\operatorname{Re} \mathbb{F}$. In the remainder of this section, we examine the structure of the complex-valued 2-form $\mathbb{F}$.

The 2-form $\mathbb{F}$ is polarized

$$
* \mathbb{F}= \pm i \mathbb{F}
$$

(cf. Definition 8.2) and degenerate

$$
\operatorname{det} \mathbb{F}=0 .
$$

It is known (see Appendix A 3) that such a 2-form is equivalent, modulo sign, to a spinor field, which is, effectively, the square root of $\mathbb{F}$. This spinor field is undotted, $\xi=\xi^{a}$, in the left-handed case [lower sign in (10.5)] and dotted, $\eta=\eta_{\dot{a}}$, in the right-handed case [upper sign in (10.5)].

Theorem 10.1. The spinor field $\xi$ associated with a left-handed massless solution satisfies the massless Dirac equation,

$$
\sigma_{a b}^{\alpha} \partial_{x^{\alpha}} \xi^{b}=0
$$

The spinor field $\eta$ associated with a right-handed massless solution satisfies the massless Dirac equation,

$$
\sigma^{\alpha \dot{b} a} \partial_{x^{\alpha}} \eta_{\dot{b}}=0
$$

Proof. It is sufficient to establish identities (10.6) and (10.7) in one coordinate system, so let us work in the coordinate system in which we have (8.7). Plugging (8.7) into (10.4), we get

$$
\mathbb{F}_{\alpha \beta}=-\frac{i a}{2}\left(\begin{array}{cccc}
0 & 0 & -1 & -1 \\
0 & 0 & \pm i & \pm i \\
1 & \mp i & 0 & 0 \\
1 & \mp i & 0 & 0
\end{array}\right) e^{i\left(x^{3}+x^{4}\right)}
$$

where the upper/lower sign corresponds to right-/left-handedness respectively. Using formulas from Appendix A 3, we conclude that

$$
\begin{aligned}
& \xi^{a}= \pm \sqrt{\frac{a}{2}}\left(\begin{array}{l}
0 \\
i
\end{array}\right) e^{i\left(x^{3}+x^{4}\right) / 2}, \\
& \eta_{\dot{a}}= \pm \sqrt{\frac{a}{2}}\left(\begin{array}{l}
1 \\
0
\end{array}\right) e^{i\left(x^{3}+x^{4}\right) / 2} .
\end{aligned}
$$

It remains only to substitute (A1) and (10.8) into (10.6), and (A2) and (10.9) into (10.7). 


\section{MASSIVE DIRAC EQUATION}

Let the diffeomorphism $\varphi$ be a massive solution as per Theorem 9.1. The corresponding deformation gradient reads

$$
D_{\beta}^{\alpha}=\delta_{\beta}^{\alpha}+\operatorname{Re}\left[i u^{\alpha} p_{\beta} e^{i p_{\gamma} x^{y}}\right]+v^{\alpha} p_{\beta} .
$$

In a particular coordinate system, the above formula turns to (9.8). Explicit calculations show that (10.1) admits a polar decomposition if and only if $c<4$. Assuming that $c<4$ and arguing as in Sec. X, we arrive at the following explicit formula for the rotation 2-form:

$$
\begin{aligned}
F & =-\frac{1}{\sqrt{c}} \operatorname{arctanh}\left(\frac{\sqrt{c}}{2}\right)\left(\operatorname{Re}\left[i\left(p \wedge u^{b}\right) e^{i p_{\gamma} x^{y}}\right]+\left(p \wedge v^{b}\right)\right) \\
& =-\frac{1}{\sqrt{c}} \operatorname{arctanh}\left(\frac{\sqrt{c}}{2}\right) \mathrm{d} A^{b}
\end{aligned}
$$

Observe that unlike the massless case (10.3), the prefactor in the RHS of (11.2) brings about, effectively, contributions nonlinear in $A$ [see (9.4)]. However, apart from the prefactor, formula (11.2) is quite simple. Here, the underlying reason is the same as in the massless case: at any given point of $\mathbb{M}$, one can identify a two-dimensional invariant subspace of the tangent fiber in which the deformation gradient (11.1) differs from the identity map.

Put

$$
\mathbb{F}:=-\frac{1}{\sqrt{c}} \operatorname{arctanh}\left(\frac{\sqrt{c}}{2}\right) \mathrm{d} \mathbb{A}^{b}=-\frac{i}{\sqrt{c}} \operatorname{arctanh}\left(\frac{\sqrt{c}}{2}\right)\left(p \wedge u^{b}\right) e^{i p_{y} x^{y}},
$$

which captures information about the oscillating part of $F$. As in Sec. X, we will now examine the geometric content of $\mathbb{F}$.

Unlike the massless case, $\mathbb{F}$ is not polarized. However, it can be decomposed into a sum of polarized pieces,

$$
\begin{gathered}
\mathbb{F}=\mathbb{F}_{+}+\mathbb{F}_{-}, \\
\mathbb{F}_{+}=\frac{\mathbb{F}-i * \mathbb{F}}{2}, \quad \mathbb{F}_{-}=\frac{\mathbb{F}+i * \mathbb{F}}{2} \\
\star \mathbb{F}_{ \pm}= \pm i \mathbb{F}_{ \pm} .
\end{gathered}
$$

In our case, the two polarized pieces are degenerate, i.e.,

$$
\operatorname{det} \mathbb{F}_{ \pm}=0
$$

The latter follows easily from the observation that the pair of identities (11.4) is equivalent to

$$
\operatorname{det} \mathbb{F}=0, \quad \mathbb{F}_{\alpha \beta} \mathbb{F}^{\alpha \beta}=0 .
$$

The 2 -form $\mathbb{F}_{-}$is equivalent, modulo sign, to an undotted spinor field $\xi=\xi^{a}$, and the 2 -form $\mathbb{F}_{+}$is equivalent, modulo sign, to a dotted spinor field $\eta=\eta_{\dot{a}}$. Since, in our case, the scalar $\xi^{a} \bar{\eta}_{a}$ is real and nonzero, one can choose the relative sign of $\xi$ and $\eta$ so that $\xi^{a} \bar{\eta}_{a}>0$. Thus, our complex-valued 2 -form $\mathbb{F}$ is equivalent to a bispinor field $(\xi, \eta)$. This bispinor field is defined uniquely up to sign and is, effectively, the square root of $\mathbb{F}$.

Theorem 11.1. The bispinor field $(\xi, \eta)$ associated with a massive solution satisfies the massive Dirac equation,

$$
-i \sigma_{a b}^{\alpha} \partial_{x^{\alpha}} \xi^{b}=m \eta_{\dot{a}}, \quad-i \sigma^{\alpha \dot{b} a} \partial_{x^{\alpha}} \eta_{\dot{b}}=m \xi^{a} .
$$

Proof. Arguing along the same lines as that of Theorem 10.1 in the special coordinate system in which we have (9.5), we get

$$
\xi^{a}=\eta_{\dot{a}}= \pm \sqrt{\frac{m a}{\sqrt{c}} \operatorname{arctanh}\left(\frac{\sqrt{c}}{2}\right)}\left(\begin{array}{l}
0 \\
i
\end{array}\right) e^{i m x^{4}}
$$

The above bispinor field clearly satisfies (11.5).

Remark 11.2. In writing the massive Dirac equation (11.5), we adopted the spinor representation [cf. Ref. 31, formula (20.2)], as opposed to the standard representation [cf. Ref. 31, formulas (21.19) and (21.17)]. 


\section{ACKNOWLEDGMENTS}

We are grateful to Z. Avetisyan, C. G. Böhmer, C. Dappiaggi, E. R. Johnson, M. Levitin, N. Saveliev, and E. Shargorodsky for valuable suggestions and stimulating discussions.

D.V. was supported by EPSRC Grant No. EP/M000079/1.

\section{APPENDIX A: NOTATION AND CONVENTIONS}

\section{Exterior calculus}

In this paper, we identify differential forms with covariant antisymmetric tensors. Henceforth, $M$ is a 4-manifold equipped with Lorentzian metric $g$ and Levi-Civita connection $\nabla$.

It is well known that the metric $g$ induces a canonical isomorphism between the tangent bundle $T M$ and the contangent bundle $T^{*} M$, the so-called musical isomorphism. We denote it by $b: T M \rightarrow T^{*} M$ and its inverse by $\sharp: T^{*} M \rightarrow T M$.

Given a scalar field $f \in C^{\infty}(M)$, its exterior derivative $\mathrm{d} f$ is defined as the gradient. Given a 1 -form $A \in \Omega^{1}(M)$, its exterior derivative $\mathrm{d} A \in \Omega^{2}(M)$ is defined, componentwise, as

$$
(\mathrm{d} A)_{\alpha \beta}=\partial_{x^{\alpha}} A_{\beta}-\partial_{x^{\beta}} A_{\alpha} .
$$

Given a pair of rank $k$ covariant antisymmetric tensors $Q$ and $T$, we define their pointwise inner product as

$$
\langle Q, T\rangle_{g}:=\frac{1}{k !} \bar{Q}_{\alpha_{1} \ldots \alpha_{k}} T_{\beta_{1} \ldots \beta_{k}} g^{\alpha_{1} \beta_{1}} \cdots g^{\alpha_{k} \beta_{k}},
$$

and, accordingly,

$$
\|Q\|_{g}^{2}:=\langle Q, Q\rangle_{g}
$$

We define the $L^{2}$ inner product,

$$
(Q, T)_{L^{2}}:=\int\langle Q, T\rangle_{g} \sqrt{-\operatorname{det} g_{\mu v}} d x
$$

Given $U \in \Omega^{k}(M)$ and $V \in \Omega^{k-1}(M)$, we define the action of the codifferential $\delta: \Omega^{k}(M) \rightarrow \Omega^{k-1}(M)$ in accordance with

$$
\langle U, \mathrm{~d} V\rangle=\langle\delta U, V\rangle
$$

In particular, when $A \in \Omega^{1}(M)$ and $F \in \Omega^{2}(M)$, we get, in local coordinates,

$$
\begin{gathered}
\delta A=-\nabla^{\alpha} A_{\alpha}, \\
(\delta F)_{\alpha}=\nabla^{\beta} F_{\alpha \beta} .
\end{gathered}
$$

For the sake of clarity, let us mention that the wedge product of 1-forms reads

$$
(A \wedge B)_{\alpha \beta}=A_{\alpha} B_{\beta}-A_{\beta} B_{\alpha}
$$

We define the action of the Hodge star on a rank $k$ antisymmetric tensor as

$$
(* Q)_{\mu_{k+1} \ldots \mu_{4}}:=\frac{1}{k !} \sqrt{-\operatorname{det} g_{\alpha \beta}} Q^{\mu_{1} \ldots \mu_{k}} \varepsilon_{\mu_{1} \ldots \mu_{4}},
$$

where $\varepsilon$ is the totally antisymmetric symbol, $\varepsilon_{1234}:=+1$.

\section{Spinors}

In this appendix and in Appendix A 3, we restrict ourselves to the special case of Minkowski space $\mathbb{M}$. We work with 2-component Weyl spinors as opposed to 4-component Dirac spinors. We recall below the basic ideas and conventions, referring the reader to Ref. 31 (Sec. 18) and Ref. 32 (Sec. 1.2) for further details.

In line with Refs. 31 and 32, we treat spinors as holonomic objects. This approach simplifies analysis in the case of flat space and is traditionally used in particle physics.

We adopt the following conventions: 
- "Metric" spinor:

$$
\epsilon_{a b}=\epsilon_{\dot{a} \dot{b}}=\epsilon^{a b}=\epsilon^{\dot{a} \dot{b}}=\left(\begin{array}{cc}
0 & 1 \\
-1 & 0
\end{array}\right) .
$$

- "Covariant," with respect to spinor indices, Pauli matrices:

$$
\sigma_{\dot{a} b}^{1}:=\left(\begin{array}{cc}
0 & 1 \\
1 & 0
\end{array}\right), \quad \sigma_{\dot{a} b}^{2}:=\left(\begin{array}{cc}
0 & -i \\
i & 0
\end{array}\right), \quad \sigma_{\dot{a} b}^{3}:=\left(\begin{array}{cc}
1 & 0 \\
0 & -1
\end{array}\right), \quad \sigma_{\dot{a} b}^{4}:=\left(\begin{array}{ll}
1 & 0 \\
0 & 1
\end{array}\right) .
$$

- "Contravariant," with respect to spinor indices, Pauli matrices:

$$
\sigma^{1 \dot{a} b}=\left(\begin{array}{cc}
0 & -1 \\
-1 & 0
\end{array}\right), \quad \sigma^{2 \dot{a} b}=\left(\begin{array}{cc}
0 & -i \\
i & 0
\end{array}\right), \quad \sigma^{3 \dot{a} b}=\left(\begin{array}{cc}
-1 & 0 \\
0 & 1
\end{array}\right), \quad \sigma^{4 a \dot{a} b}=\left(\begin{array}{ll}
1 & 0 \\
0 & 1
\end{array}\right) .
$$

Here, $\sigma^{\alpha \dot{a} b}=\epsilon^{\dot{a} \dot{c}} \epsilon^{b d} \sigma^{\alpha}{ }_{i d}$.

Pauli matrices satisfy the identities

$$
\begin{aligned}
& \sigma^{\alpha \dot{b} a} \sigma^{\beta}{ }_{b c}+\sigma^{\beta \dot{b} a} \sigma^{\alpha}{ }_{b c}=-2 g^{\alpha \beta} \delta^{a}{ }_{c}, \\
& \sigma_{a b b}^{\alpha} \sigma^{\beta c b}+\sigma^{\beta}{ }_{\dot{a} b} \sigma^{\alpha \dot{c} b}=-2 g^{\alpha \beta} \delta_{\dot{a}}{ }^{c} .
\end{aligned}
$$

\section{Spinor representation of 2-forms}

Let $\mathbb{F}_{-}$and $\mathbb{F}_{+}$be polarized complex 2-forms [see (11.3)]. Then, $\mathbb{F}_{-}$is equivalent to a trace-free undotted rank two spinor $\zeta_{c}^{b}$,

$$
\left(\mathbb{F}_{-}\right)^{\alpha \beta}=-i \sigma^{\alpha}{ }_{a b} \zeta_{c}^{b} \sigma^{\beta a c},
$$

and $\mathbb{F}_{+}$is equivalent to a trace-free dotted rank two spinor $\theta_{\dot{b}}{ }^{c}$,

$$
\left(\mathbb{F}_{+}\right)^{\alpha \beta}=i \sigma^{\alpha \dot{b a}} \theta_{\dot{b}}^{\dot{c}} \sigma^{\beta} \dot{c} .
$$

The identities (A3a) and (A3b) ensure that the right-hand sides of (A4a) and (A4b), respectively, are antisymmetric in $\alpha, \beta$.

Fact A.3.1. The following are equivalent:

(i) $\operatorname{det} \mathbb{F}_{-}=0$.

(ii) $\operatorname{det} \zeta=0$.

(iii) There exists a rank one spinor $\xi^{a}$ such that $\zeta_{c}^{b}=\xi^{b} \xi^{d} \epsilon_{d c}$.

Fact A.3.2. The following are equivalent:

(i) $\operatorname{det} \mathbb{F}_{+}=0$.

(ii) $\operatorname{det} \theta=0$.

(iii) There exists a rank one spinor $\eta_{\dot{a}}$ such that $\theta_{\dot{b}}{ }^{\dot{c}}=\eta_{\dot{b}} \eta_{\dot{d}} \epsilon^{\dot{d} \dot{c}}$.

Facts A.3.1 and A.3.2 imply that a degenerate polarized 2-form is equivalent to the square of a rank 1 spinor. The latter is defined uniquely up to sign.

The equivalence between (i) and (ii) in the above statements is a straightforward consequence of (A4a) and (A4b), whereas (iii) is not so obvious. The relevant arguments are presented in Appendix B 2.

\section{APPENDIX B: SOME RESULTS IN LINEAR ALGEBRA}

\section{Linear algebra involving a pair of quadratic forms}

Working in an $n$-dimensional real vector space $V$, consider a pair of non-degenerate symmetric bilinear forms, $g: V \times V \rightarrow \mathbb{R}$ and $h: V \times V \rightarrow \mathbb{R}$. These uniquely define an invertible linear operator $L: V \rightarrow V$ via the formula

$$
h(u, v)=g(L u, v), \quad \forall u, v \in V .
$$


The eigenvalue problem for the operator $L$,

$$
L u=\lambda u
$$

can be equivalently reformulated in terms of bilinear forms

$$
h(u, v)=\lambda g(u, v), \quad \forall v \in V .
$$

The expression $h-\lambda g$ is called a linear pencil of symmetric bilinear forms.

It is well known [Ref. 33, Sec. X.6] that if at least one of the forms is sign definite, then $L$ has real eigenvalues and is diagonalizable. In this case, the associated pencil is called regular.

If neither $g$ nor $h$ is sign definite, then the operator $L$ may have complex eigenvalues and may not be diagonalizable. In particular, the strain operator

$$
S:=L-\mathrm{Id}
$$

may be nilpotent. This is a fundamental difference with the regular (sign definite) case where the strain operator cannot be nilpotent.

We now address the question what is the maximal nilpotency index of $S$.

Lemma B.1. Suppose that $n \geq 4$ and that both $g$ and $h$ have Lorentzian signature,

$$
\underbrace{+\cdots+}_{n-1}-
$$

Then, the nilpotency index of $S$ is less than or equal to 3.

Proof. Observe first that it is sufficient to prove the lemma in the complex setting, where we can use Theorem 8.4.1 (Ref. 34). Examination of the latter shows that the nilpotency index strictly greater than 4 is not possible, whereas the nilpotency index equal to 4 is possible only if we have an invariant subspace in which our operator has the structure similar to formula (8.4.19) of Ref. 34. However, the matrix $N$ from formula (8.4.19) (Ref. 34) with $\lambda=0$ has the nilpotency index at most 3.

Remark B.2. Closer examination shows that in our setting, the structure similar to formula (8.4.19) of Ref. 34 cannot be realized because the latter describes an operator that is Lorentz-normal but not Lorentz-symmetric. The only way the strain operator can get nilpotency index 3 is when it has a Jordan block of the type similar to formula (8.4.18) of Ref. 34 with $\lambda=r=0$. As a final observation, let us point out that in dimensions $n=2$ and $n=3$, the maximal nilpotency indices 2 and 3 can actually be attained.

\section{Nilpotent operators in a 2D symplectic space}

Lemma B.3. Let $V$ be a two-dimensional complex vector space equipped with a symplectic form $\omega$, and let $L: V \rightarrow V$ be a linear operator. Then, $L$ is nilpotent if and only if there exists a $u \in V$ such that

$$
L v=u \omega(u, v), \quad \forall v \in V .
$$

Proof. An operator of the form (B1) is clearly nilpotent. Hence, we only need to prove the converse statement.

Let $L$ be nilpotent. Choose a basis in $V$ so that the symplectic form reads

$$
\omega(v, w)=\varepsilon_{r s} v^{r} w^{s}
$$

where $\varepsilon$ is the totally antisymmetric symbol, $\varepsilon_{12}=+1$. The linear operator $L$ is represented in this basis by the matrix

$$
L_{s}^{r}=\left(\begin{array}{ll}
a & b \\
c & d
\end{array}\right) .
$$

The nilpotency condition is equivalent to the trace and the determinant of $L$ both being 0 . Hence, (B3) can be rewritten as

$$
L_{s}^{r}=\left(\begin{array}{cc}
\sqrt{-b c} & b \\
c & -\sqrt{-b c}
\end{array}\right)
$$

with appropriate choice of complex square root. The matrix (B4) can be factorized as

$$
L_{s}^{r}=\left(\begin{array}{c}
\sqrt{b} \\
-\sqrt{-c}
\end{array}\right)\left(\begin{array}{ll}
\sqrt{b} & -\sqrt{-c}
\end{array}\right)\left(\begin{array}{cc}
0 & 1 \\
-1 & 0
\end{array}\right)
$$


where the square roots are chosen in such a way that $\sqrt{b} \sqrt{-c}=\sqrt{-b c}$. Formulas (B5) and (B2) give us (B1) with

$$
u=\left(\begin{array}{c}
\sqrt{b} \\
-\sqrt{-c}
\end{array}\right)
$$

\section{APPENDIX C: DIFFERENTIAL GEOMETRIC CHARACTERIZATION OF SCREW GROUPS}

Let $S G$ be one of the screw groups $\mathrm{SG}_{0}^{+}, \mathrm{SG}_{0}^{-}$, or $\mathrm{SG}_{m}$ defined in Sec. VII. In what follows, the (global) isomorphism $T \mathbb{M} \simeq \mathbb{M} \times \mathbb{M}$ will be tacitly understood. In particular, we will not distinguish between points of $M$ and vectors in the tangent fibers.

Direct inspection shows that for any $P, Q \in \mathbb{M}$, there exists a unique $\xi \in \mathrm{SG}$ such that $\xi(P)=Q$. This allows us to define a map

$$
\begin{gathered}
\Upsilon: T_{P} \mathbb{M} \rightarrow T_{Q} \mathbb{M}, \\
V \mapsto \xi(P+V)-Q,
\end{gathered}
$$

depending only on $P$ and $Q$, which, in turn, determines $\xi$. The map $\Upsilon$ is linear and defines a metric compatible affine connection with vanishing curvature and nonvanishing torsion. Such connections are known as Weitzenböck (teleparallel) connections.

We define the covariant derivative of a vector field as

$$
\frac{\partial v^{\alpha}}{\partial x^{\beta}}+\Upsilon_{\beta \gamma}^{\alpha} v^{y}
$$

and torsion as

$$
T_{\beta \gamma}^{\alpha}:=\Upsilon_{\beta \gamma}^{\alpha}-\Upsilon_{\gamma \beta}^{\alpha} .
$$

It is known [Ref. 35, formula (7.34)] that a metric compatible affine connection is determined by metric and torsion, so torsion provides a convenient tensorial description of a connection.

Torsion has three irreducible pieces [Ref. 36, formulas (4.1)-(4.4)],

$$
\begin{gathered}
T=T^{\mathrm{ax}}+T^{\mathrm{vec}}+T^{\mathrm{ten}}, \\
T_{\alpha \beta \gamma}^{\mathrm{ax}}=\frac{1}{3}\left(T_{\alpha \beta \gamma}+T_{\beta \gamma \alpha}+T_{\gamma \alpha \beta}\right), \\
T_{\alpha \beta \gamma}^{\mathrm{vec}}=\frac{1}{3}\left(g_{\alpha \beta} T^{\mu}{ }_{\mu \gamma}-g_{\alpha \gamma} T^{\mu}{ }_{\mu \beta}\right),
\end{gathered}
$$

labeled by the adjectives axial, vector, and tensor, respectively. We remind the reader that we raise and lower tensor indices using the metric $g$.

Lemma C.1. For all three groups, $\mathrm{SG}_{0}^{+}, \mathrm{SG}_{0}^{-}$, and $\mathrm{SG}_{m}$ torsion is constant, and vector torsion is zero. The corresponding formulas for axial torsion read

$$
\begin{aligned}
& \left(* T_{ \pm}^{\mathrm{ax}}\right)_{\alpha}=\mp \frac{2}{3}(0,0,1,1), \\
& \left(* T_{m}^{\mathrm{ax}}\right)_{\alpha}=-\frac{4}{3}(0,0, m, 0) .
\end{aligned}
$$

Proof. Straightforward calculations give the following expressions for the nonzero connection coefficients:

- For $\mathrm{SG}_{0}^{ \pm}$,

$$
\begin{array}{ll}
\Upsilon_{32}^{1}= \pm 1, & \Upsilon_{31}^{2}=\mp 1, \\
\Upsilon_{42}^{1}= \pm 1, & \Upsilon_{41}^{2}=\mp 1 .
\end{array}
$$

- For $\mathrm{SG}_{m}$,

$$
\Upsilon_{42}^{1}=2 m, \quad \Upsilon_{41}^{2}=-2 m .
$$

It remains only to substitute the above expressions into formulas (C1)-(C3). 


\section{APPENDIX D: EXPLICIT FORMULAS FOR OUR FIELD EQUATIONS}

In this appendix, we sketch out an algorithm for the derivation of the explicit form of the differential operator $E(\varphi)$ introduced in Sec. III. We will do this for the special case of a Lagrangian of the form (2.9) from Example 2.3 and in Minkowski space. Throughout this appendix, we shall use the notation $\partial_{\alpha}=\partial / \partial x^{\alpha}$.

Substituting (2.9) into (2.16), we get

$$
L\left(e_{2}, e_{3}, e_{4}\right)=\alpha\left(e_{2}+e_{3}+e_{4}\right)^{2}+\beta e_{2}
$$

To begin with, let us rewrite the scalars $e_{3}$ and $e_{4}$ in terms of $\operatorname{tr}\left(S^{k}\right), k=1,2,3,4$,

$$
\begin{aligned}
& e_{3}=\frac{1}{6}\left[(\operatorname{tr} S)^{3}-3(\operatorname{tr} S) \operatorname{tr}\left(S^{2}\right)+2 \operatorname{tr}\left(S^{3}\right)\right] \\
& e_{4}=\frac{1}{24}\left[(\operatorname{tr} S)^{4}-6(\operatorname{tr} S)^{2} \operatorname{tr}\left(S^{2}\right)+3\left(\operatorname{tr}\left(S^{2}\right)\right)^{2}+8(\operatorname{tr} S) \operatorname{tr}\left(S^{3}\right)-6 \operatorname{tr}\left(S^{4}\right)\right] .
\end{aligned}
$$

Substituting (2.5b), (D2a), and (D2b) into (D1), we get a representation of our Lagrangian $L$ as a linear combination of terms

$$
\prod_{j=1}^{k} S^{\alpha_{j}} \beta_{j},
$$

where $\left\{\beta_{1}, \ldots, \beta_{k}\right\}$ is some permutation of $\left\{\alpha_{1}, \ldots, \alpha_{k}\right\}$. The number $k$ takes values from 2 to 8 . In what follows, we write down the contribution to $E(\varphi)$ coming from a single term (D3).

The explicit formula for the strain tensor reads

$$
S_{\beta}^{\alpha}=\partial_{\beta} A^{\alpha}+\partial^{\alpha} A_{\beta}+\left(\partial^{\alpha} A_{\gamma}\right)\left(\partial_{\beta} A^{\gamma}\right)
$$

Variation $A^{\alpha}(x) \mapsto A^{\alpha}(x)+\Delta A^{\alpha}(x)$ gives us

$$
\begin{aligned}
\Delta S_{\beta}^{\alpha}=\partial_{\beta}\left(\Delta A^{\alpha}\right) & +\partial^{\alpha}\left(\Delta A_{\beta}\right)+\left(\partial^{\alpha}\left(\Delta A_{\gamma}\right)\right)\left(\partial_{\beta} A^{\gamma}\right)+\left(\partial^{\alpha} A_{\gamma}\right)\left(\partial_{\beta}\left(\Delta A^{\gamma}\right)\right) \\
& =\delta^{\alpha}{ }_{\gamma} \partial_{\beta}\left(\Delta A^{\gamma}\right)+g_{\beta \gamma} \partial^{\alpha}\left(\Delta A^{\gamma}\right)+\left(\partial^{\alpha}\left(\Delta A^{\gamma}\right)\right)\left(\partial_{\beta} A_{\gamma}\right)+\left(\partial^{\alpha} A_{\gamma}\right)\left(\partial_{\beta}\left(\Delta A^{\gamma}\right)\right) .
\end{aligned}
$$

We define the linear differential operator

$$
D_{\beta \gamma}^{\alpha}:=\left[g_{\beta \gamma}+\left(\partial_{\beta} A_{\gamma}\right)\right] \partial^{\alpha}+\left[\delta_{\gamma}^{\alpha}+\left(\partial^{\alpha} A_{\gamma}\right)\right] \partial_{\beta}+2\left(\partial^{\alpha} \partial_{\beta} A_{\gamma}\right) .
$$

The contribution to $E(\varphi)$ coming from (D3) reads

$$
-\sum_{l=1}^{k} D^{\alpha_{l}} \beta_{l} \gamma \prod_{\substack{j=1 \\ j \neq l}}^{k} S^{\alpha_{j}} \beta_{j} .
$$

The above algorithm can be easily generalized to spacetimes with $x$-dependent metric and to Lagrangians of general form.

\section{REFERENCES}

${ }^{1}$ P. G. Ciarlet, Mathematical Elasticity: Three-Dimensional Elasticity, Volume 1, Studies in Mathematics and its Applications Vol. 20 (North-Holland, Amsterdam, 1988).

${ }^{2}$ P. G. Ciarlet, An Introduction to Differential Geometry with Applications to Elasticity (Springer Netherlands, 2005).

${ }^{3}$ M. Capoferri, N. Saveliev, and D. Vassiliev, "Classification of first order sesquilinear forms," Rev. Math. Phys. 32, 2050027 (2020).

${ }^{4}$ L. D. Landau and E. M. Lifshitz, Theory of Elasticity, Course of Theoretical Physics Vol. 7, 3rd ed. (Pergamon, Oxford, 1986), translated from the Russian by J. B. Sykes and W. H. Reid.

${ }^{\mathbf{5}}$ A.-L. Cauchy, “Sur les équations qui expriment les conditions d'équilibre ou les lois du mouvement intérieur d'un corps solide, élastique ou non élastique," Exercices Math. 3, 160-187 (1828), also in Oeuvres Complètes Ser. II 8, 195-226.

${ }^{6}$ A.-L. Cauchy, “Sur l'équilibre et le mouvement intérieur des corps considérés commes des masses continues," Exercices Math. 4, 293-319 (1829), also in Oeuvres Complètes Ser. II 9, 342-369.

${ }^{7}$ A. J. M. Spencer, Continuum Mechanics (Dover, 2004).

${ }^{8}$ J. Eells and J. H. Sampson, "Harmonic mappings of Riemannian manifolds," Am. J. Math. 86, 109-160 (1964).

${ }^{9}$ P. Baird and J. C. Wood, Harmonic Morphisms Between Riemannian Manifolds, London Mathematical Society Monographs Vol. 29 (Oxford University Press, 2003).

${ }^{10}$ N. Rosen, "General relativity and flat space I," Phys. Rev. 57, 147-150 (1940).

${ }^{11}$ C. de Rham, G. Gabadadze, and A. J. Tolley, "Resummation of massive gravity," Phys. Rev. Lett. 106, 231101 (2011).

${ }^{12}$ S. F. Hassan and R. A. Rosen, “Bimetric gravity from ghost-free massive gravity," J. High Energy Phys. 2012(2), 126. 
${ }^{13}$ A. Schmidt-May and M. von Strauss, “Recent developments in bimetric theory,” J. Phys. A: Math. Theor. 49, 183001 (2016).

${ }^{14}$ J. Jost, Riemannian Geometry and Geometric Analysis (Springer-Verlag, 2011).

${ }^{15}$ W. S. Ożański, “The Lagrange multiplier and the stationary Stokes equations,” J. Appl. Anal. 23(2), 137-140 (2017).

${ }^{16}$ J. L. Synge, Relativity: The General Theory (North-Holland Publishing Company, Amsterdam, 1960).

${ }^{17}$ C. Truesdell and W. Noll, The Non-Linear Field Theories of Mechanics, 3rd ed. (Springer-Verlag Berlin Heidelberg, 2004).

${ }^{18}$ Y. Bolshakov, C. V. M. van der Mee, A. C. M. Ran, B. Reichstein, and L. Rodman, "Polar decompositions in finite dimensional indefinite scalar product spaces: General theory," Linear Algebra Appl. 261, 91-141 (1997).

${ }^{19}$ C. Mehl, A. C. M. Ran, and L. Rodman, "Polar decompositions of normal operators in indefinite inner product spaces," in Operator Theory in Krein Spaces and Nonlinear Eigenvalue Problems, Operator Theory: Advances and Applications Vol. 162, edited by K. H. Förster, P. Jonas, and H. Langer (Birkhäuser Basel, 2005).

${ }^{20}$ J. Marsden, D. Ebin, and A. Fisher, "Diffeomorphisms group, hydrodynamics and relativity," in Proceedings of the 13th Biennial Seminar of Canadian Mathematical Congress (Canadian Mathematical Society, Montreal, Canada, 1972), pp. 135-279.

${ }^{21}$ J. Milnor, "Remarks on infinite-dimensional Lie groups," in Relativity, Groups, and Topology II, Les Houches, edited by B. S. DeWitt and R. Stora (Elsevier, Amsterdam, 1983).

${ }^{22}$ A. Banyaga, The Structure of Classical Diffeomorphism Groups (Springer US, 1997).

${ }^{23}$ A. Kriegl and P. Michor, The Convenient Setting of Global Analysis, Mathematical Surveys and Monographs Vol. 53 (American Mathematical Society, 1997).

${ }^{24}$ Yu. Safarov and D. Vassiliev, The Asymptotic Distribution of Eigenvalues of Partial Differential Operators (American Mathematical Society, Providence, RI, 1997).

${ }^{25}$ S. Agmon, A. Douglis, and L. Nirenberg, "Estimates near the boundary for solutions of elliptic partial differential equations satisfying general boundary conditions II," Commun. Pure Appl. Math. 17, 35-92 (1964).

${ }^{26}$ W. W.-Y. Wong, “Regular hyperbolicity, dominant energy condition and causality for Lagrangian theories of maps,” Classical Quantum Gravity 28, 215008 (2011).

${ }^{27}$ A. O. Barut and R. Raczka, Theory of Group Representations and Applications, 2nd ed. (World Scientific Publishing Co., Singapore, 1986).

${ }^{28}$ E. Cartan, The Theory of Spinors (Dover Publications, Inc., 2003), reprint of the 1981 English translation.

${ }^{29}$ M. Born and L. Infeld, "Foundations of the new field theory," Proc. R. Soc. London, Ser. A 144, 425-451 (1934).

${ }^{30}$ J. B. Jiménez, L. Heisenberg, G. J. Olmo, and D. Rubiera-Garcia, “Born-Infeld inspired modifications of gravity,” Phys. Rep. 727, 1-129 (2018).

${ }^{31}$ V. B. Berestetskii, E. M. Lifshitz, and L. P. Pitaevskii, Quantum Electrodynamics, Course of Theoretical Physics Vol. 4, 2nd ed. (Pergamon Press, 1982).

${ }^{32}$ I. L. Buchbinder and S. M. Kuzenko, Ideas and Methods of Supersymmetry and Supergravity, Revised edition (Taylor \& Francis, 1998).

${ }^{33}$ F. R. Gantmacher, The Theory of Matrices, Volume 1 (Chelsea Publishing Company, 1959).

${ }^{34}$ I. Gohberg, P. Lancaster, and L. Rodman, Indefinite Linear Algebra and Applications (Birkhäuser, Basel, 2005).

${ }^{35}$ M. Nakahara, Geometry, Topology and Physics, 2nd ed. (CRC Press, 2003).

${ }^{36}$ J. D. McCrea, "Irreducible decompositions of non-metricity, torsion, curvature and Bianchi identities in metric-affine spacetimes," Classical Quantum Gravity 9, 553-568 (1992). 